\title{
Myxomycete diversity in the coastal desert of Peru with emphasis on the lomas formations
}

\author{
Carlos Lado ${ }^{1 *}$, Diana Wrigley de Basanta ${ }^{1}$, Arturo Estrada-Torres ${ }^{2} \&$ Steven L. Stephenson ${ }^{3}$ \\ ${ }^{1}$ Real Jardín Botánico de Madrid, CSIC. Plaza de Murillo, 2 - 28014 Madrid, Spain. Lado@rjb.csic.es, dwbasanta@gmail.com \\ ${ }^{2}$ Centro de Investigación en Ciencias Biológicas, Univ. Autónoma de Tlaxcala, Apdo. Postal 183, Tlaxcala 90000, Mexico. arturomixo@hotmail.com \\ ${ }^{3}$ Department of Biological Sciences, University of Arkansas, Fayetteville, AR 72701, U.S.A. slsteph@uark.edu
}

\begin{abstract}
Lado, C., Wrigley de Basanta, D., Estrada-Torres, A. \& Stephenson, S.L. 2016. Myxomycete diversity in the coastal desert of Peru with emphasis on the lomas formations. Anales Jard. Bot. Madrid 73(1): e032.

Results obtained from the first survey for myxomycetes in the hyperarid areas of Peru are reported. Sampling over three consecutive years was carried out at 96 localities between 0 and 1500 m elevation. A total of 78 species from 23 genera in all 6 myxomycete orders were determined from 723 collections of myxomycetes. One new species, Didymium peruvianum, is described, 2 species new to the Neotropics, 4 new records for South America and 41 additional new species for Peru are reported, increasing the country catalogue by over $50 \%$. Results show that arid areas are rich in myxomycetes, and that each area has a unique species assemblage. Endemic plants had a particular relevance as myxomycete substrates. The predominance of the order Physarales in arid areas is reinforced, and the ecological importance of coastal fogs (garúas) is evident from the results. Comments are included on interesting or rare collections, as are SEM micrographs of several species and statistical evaluation of the results.
\end{abstract}

Keywords: Amoebozoa, arid ecoregion, distribution, endemic plants, Eumycetozoa, myxobiota, Myxogastria, plasmodial slime moulds.

\section{INTRODUCTION}

The Myxomycetes or plasmodial slime moulds are a group of microorganisms included in the Amoebozoa, a major taxonomic group of amoeboid protists, considered as a protozoan fungal analogue (Kirk \& al., 2011) due to their characteristic of producing spores in static fruiting bodies. Myxomycetes have been found in all terrestrial ecosystems, and have, until recently, been associated with temperate humid environments, on account of the need for water to complete their life cycle. However it has become evident that an exclusive and interesting number of this myxobiota, thrive in extremely arid or semi-arid environments, such as warm and cold deserts where the availability of liquid water from rain is practically nil. The number of species in these arid regions, especially in the Neotropics, is surprisingly high, since in the Atacama Desert 24 species were recorded by Lado \& al. (2007), more than 100 taxa were reported by Estrada-Torres \& al. (2009) in the deserts of Mexico, and in the Monte desert of Argentina 72 species were isolated (Lado \& al., 2011). Research carried out in the Patagonian steppe registered 133 different

\section{Resumen}

Lado, C., Wrigley de Basanta, D., Estrada-Torres, A. \& Stephenson, S.L. 2016. La diversidad de Myxomycetes en el desierto costero de Perú con especial atención a las formaciones de lomas. Anales Jard. Bot. Madrid 73(1): e032.

Se dan a conocer los resultados del primer estudio realizado sobre los Myxomycetes de las regiones hiperáridas de Perú. El muestreo, realizado en tres años consecutivos, se ha llevado a cabo en 96 localidades situadas entre 0 y $1500 \mathrm{~m}$ de altitud. En total se obtuvieron 723 colecciones de mixomicetes, pertenecientes a 6 órdenes, 23 géneros y 78 especies. Se describe una nueva especie, Didymium peruvianum, 2 especies se citan por primera vez en el Neotrópico, 4 son nuevos registros para América del Sur y 41 especies más son nuevos registros para Perú, con lo que el catálogo del país se incrementa en un $50 \%$. Los resultados muestran que las zonas áridas son ricas en mixomicetes, y que cada area tiene un conjunto único de especies. Las plantas endémicas, dominantes en estos ambientes, tuvieron una relevancia especial como sustrato para los myxomycetes. Se pone de manifiesto el predominio de los representantes del órden Physarales en las zonas áridas, lo que refuerza resultados previos, así como la importancia ecológica de las nieblas costeras (garúas). Se incluyen comentarios de las especies más interesantes o poco comunes, así como fotografías realizadas con microscopio electrónico de barrido, y una evaluación estadística de los resultados.

Palabras clave: Amoebozoa, distribución, ecorregión árida, Eumycetozoa, hongos mucilaginosos plasmodiales, myxobiota, Myxogastria, plantas endémicas.

species (Lado \& al., 2014), almost the $15 \%$ of the total number of species known worldwide. These arid regions have become even more interesting because of the number of new species that have been discovered and described from them (Lado \& al., 1999, 2007, 2009, 2013, 2014; Estrada-Torres \& al., 2001, 2009; Wrigley de Basanta \& al., 2008b, 2009, 2010a, 2011, 2012, 2015). These new species represent four different orders, and the genera Cribraria, Didymium, Licea, Macbrideola, Perichaena and Physarum.

Since the publication of the first records by Rudolphi (1829), only 31 species of myxomycete had been published from Peru, until a recent paper increased the number to 80 (Rojas \& al., 2011), but almost all of these records were from the tropical forests of the Amazon basin, to the East of the Peruvian Andes. In the checklist compiled by Lado \& Wrigley de Basanta (2008) the country was among the least studied in the whole of the Neotropics with only five publications referring to Myxomycetes. There are no data at all from the arid areas of Peru, and no systematic study, catalogue or inventory had ever been carried out on this enormous dry Peruvian territory. A three-year study, supported by a grant from the Spanish Government, was 
undertaken to carry out a field-based investigation of the myxobiota of these arid areas. In this paper the first substantial data set on the Myxomycetes of the drylands of Peru is provided.

The ecoregion to be included in the study is the Peruvian Coastal Desert. This desert forms a continuous belt of arid land for more than $2500 \mathrm{~km}$, extending between the Pacific Ocean and the Western slopes of the Andes. It is limited to the North by the border with Ecuador $\left(3^{\circ} 25^{\prime} \mathrm{S}\right)$ and to the South by the Chilean border and the Atacama Desert $\left(18^{\circ} 21^{\prime} \mathrm{S}\right)$, and makes up about $11 \%$ of the country
(Rundel \& al., 1991). It is a hyper-arid territory, receiving $0-300 \mathrm{~mm}$ of rain annually. The coast bordering the desert to the West is bathed by the cold Humboldt Current, and subsequent temperature inversion results in dense coastal fogs or "garuas" that settle on the lower westerly slopes of the Andes, and provide the only moisture for certain plants to grow in an otherwise barren desert (Dillon, 1997). The hyper-aridity of the southern Peruvian Desert appears to be very old, at least regionally, and has largely prevailed for the last 13-15 million years. The uplift of central Andean Cordillera during the Oligocene and Early Miocene was

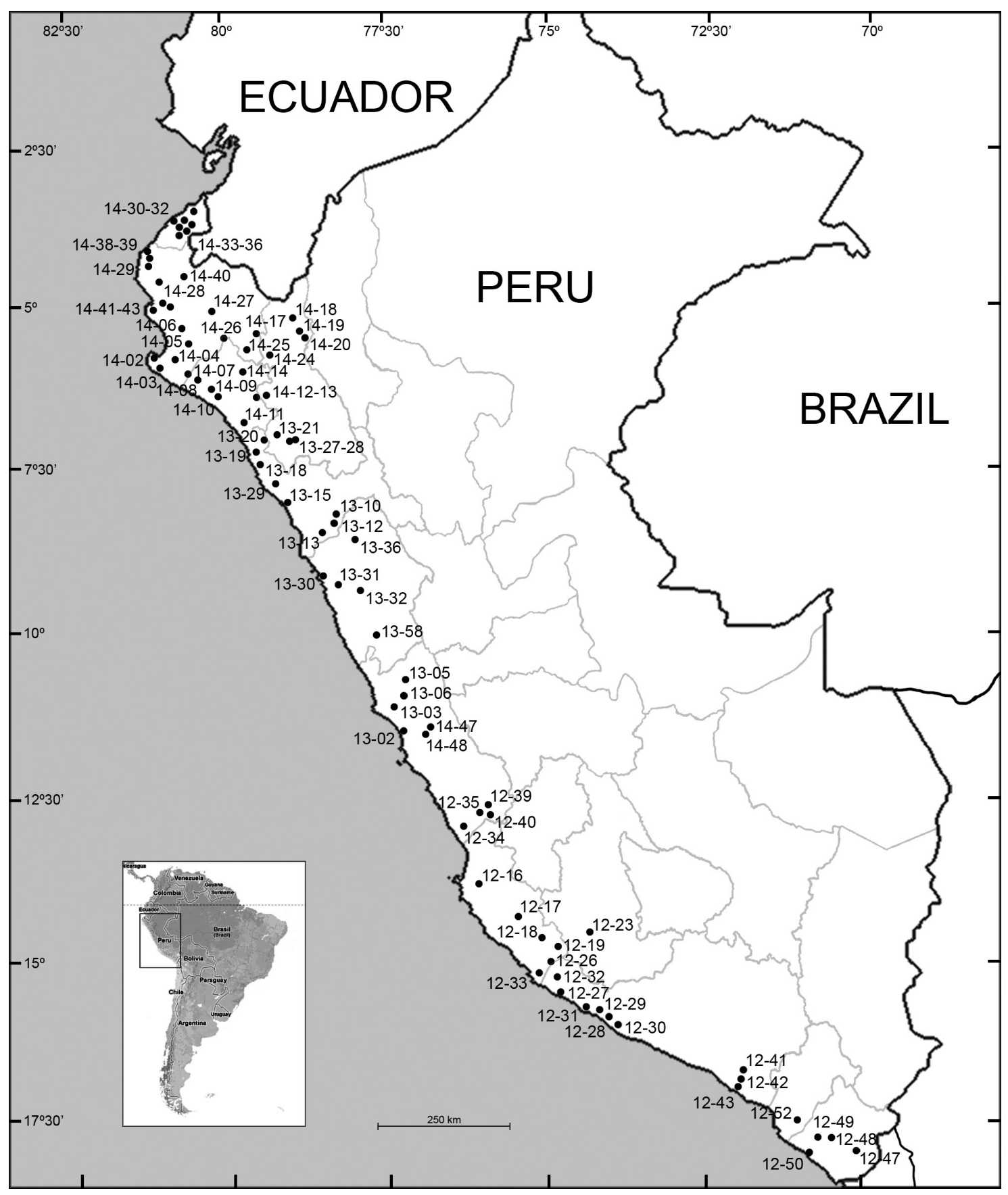

Fig. 1. Map of the general study area and localities sampled that were positive for myxomycete species (numbers refer to Table 1). 
a critical factor in the formation of these arid conditions (Rundel \& al., 1991). In the North, the Sechura Desert, sometimes has torrential rain associated with the phenomenon of El Niño, the fact that the Humboldt Current moves West away from the coast at these latitudes, and the proximity of the area is to the humid tropical weather from the Equator.

Emphasis of the study reported in this paper was placed on the lower elevations $(0-1,500 \mathrm{~m})$ that include the "lomas formations" (Weberbauer, 1945). These are terrestrial islands surrounded by hyper-arid areas devoid of plants. They cover only about $5000 \mathrm{~km}^{2}$ of this immense desert, but a recent checklist of vegetation in the lomas formations registered 847 plant species from 83 families (Dillon \& al., 2011). Some are species found in other deserts of North America, but others are endemics sometimes occurring in only a single locality. These plants are potential substrates for myxomycetes, providing rich and varied microhabitats in which these organisms can complete their development.

The study area covers the coastal areas in the administrative Departments of Tumbes, Piura, Lambayeque,
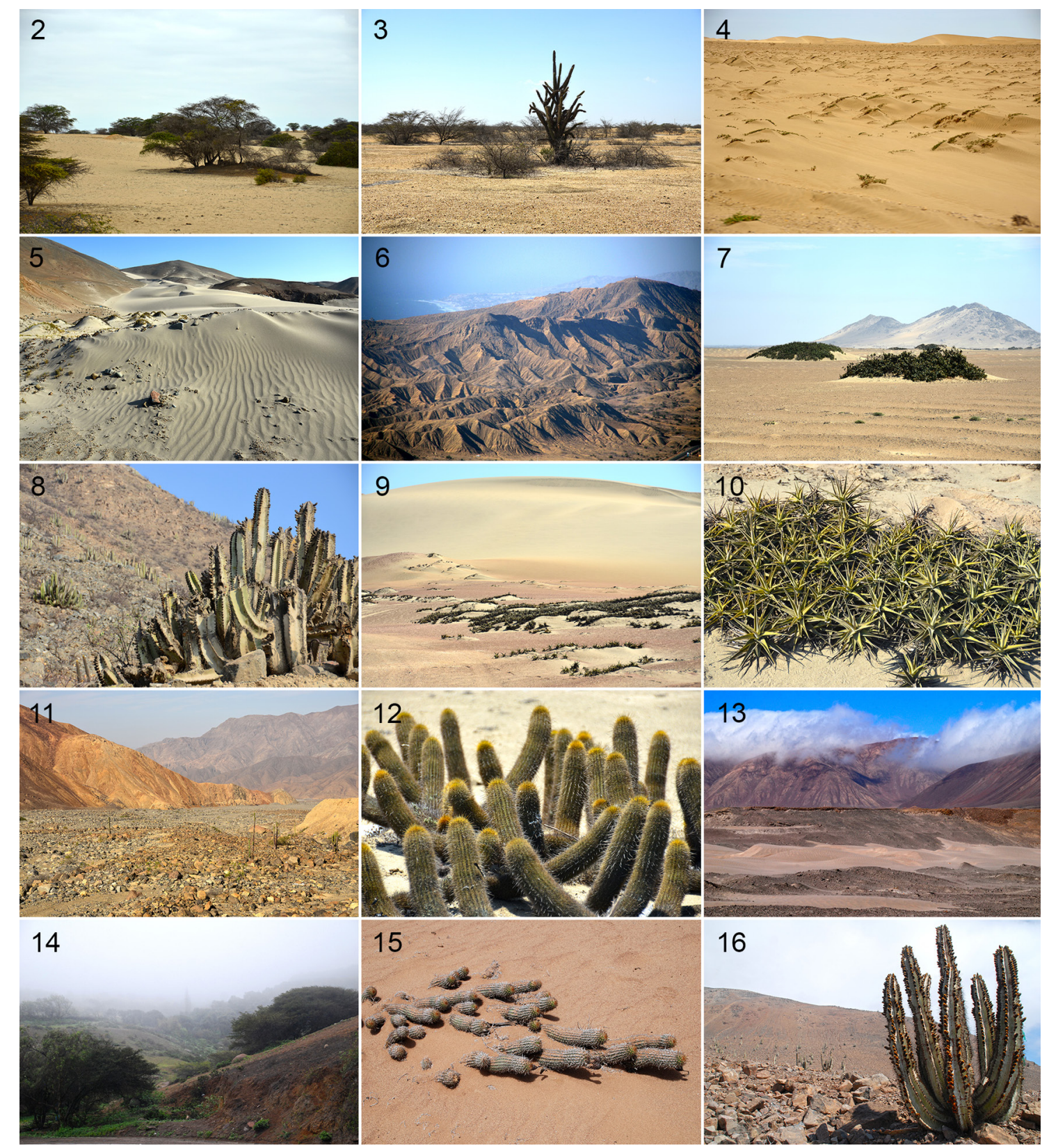

Figs. 2-16. Landscapes and some vegetation types of the coastal desert of Peru. 2-3. Trees such as Prosopis sp. and cacti in the sandy soils of Tumbes. 4-5. Flat sandy plains and active dunes in the Sechura Desert. 6. Hills near the coastal areas. 7. Dunes stabilised by species of the genus Capparis. 8. Neoraimondia sp. near Lima. 9-10. Dunes with islands of terrestrial bromeliads of the genus Tillandsia. 11. Arid valleys. 12. Haageocereus sp. near Moche, La Libertad. 13. Fog retained by the hills in the area of lomas de Atiquipa. 14. Vegetation influenced by the "garua" in the lomas de Atiquipa. 15. Haageocereus sp. in Sama Grande, Tacna. 16. Population of Neoraimondia aticensis near the coast of Sama, Tacna. 
Cajamarca, La Libertad, Ancash, Lima, Ica, Arequipa, Moquegua and Tacna (Figs. 1, 2-16). The abundance of vegetation in Tumbes and the presence of trees (Figs. 2-3) are the result of greater precipitation due to the influence of El Niño and its proximity to the tropics as mentioned above. In Piura the Sechura Desert, with extensive areas of flat sandy plains and active dunes (Figs. 4-5), is where the coastal desert spreads out to its widest and can reach up to a width of $100 \mathrm{~km}$. The dunes are stabilised by species of the genus Capparis (Fig. 7), plants that have proved to be most productive substrates for myxomycetes. Parts of the desert along courses of rivers that flow to the Pacific Ocean in Lambayeque, Cajamarca and La Libertad, have been transformed into agricultural land and planted with crops like sugar cane, asparagus or orchards of fruit trees. On the lower sandy slopes, below $200 \mathrm{~m}$, a mixture of cacti (Espostoa, Haageocereus, Melocactus) and terrestrial bromeliads of the genus Tillandsia can be found (Figs. 8-10, $12,15-16)$. On the hills and stony mountain slopes, under the influence of the "garuas" (Figs. 6, 13-14), a number of endemic species of plants can be found (Leiva González $\&$ al., 2014). In the central zone (Ancash, Lima, Ica, North of Arequipa) the coast and land below $100 \mathrm{~m}$ is devoid of vegetation, and from $100-300 \mathrm{~m}$ a community of cryptogams predominates with species of Nostoc and foliose and fruticose lichens (Fig. 11). Around $300 \mathrm{~m}$ herbaceous and woody plants appear and above $300 \mathrm{~m}$, the dry rocky slopes support stands of Tillandsia latifolia and Puya ferruginea. Most of the original vegetation around Lima has disappeared under the significant human pressure on the land. In Ica there are steep coastal ridges with a series of marine terraces. The fierce coastal winds generate enormous dunes that are stabilised in some places only by the growth of species of Tillandsia. The lomas de Atiquipa (Figs. 13-14), one of the best-preserved examples (Gómez-Sosa, 1986) and one of the richest in species, was among the areas studied in Arequipa. In the southern zone (South of Arequipa, Moquegua and Tacna) the vegetation contains sparsely distributed shrubs and herbaceous species and a population of Neoraimondia aticensis occurs near the ocean (Fig. 16). Large colonies of Tillandsia purpurea cover the sand dunes (Dillon, 1997).

\section{MATERIAL AND METHODS}

Sampling was carried out in 96 localities (Table 1), between 0 and $1500 \mathrm{~m}$ elevation of which 83 yielded identifiable myxomycetes. Work was carried out in three expeditions in September to October 2012, February to March 2013 and in May 2014. An additional site is included from a different elevation (PER-12-23), as the new species was isolated from substrate material found there, in addition to the two coastal collections. Localities were selected at intervals of approximately $25-50 \mathrm{~km}$. At each site, the microhabitats in which myxomycetes are known or suspected to occur were examined carefully, and were georeferenced with a portable GPS unit (Garmin eTrex Vista HCX, Datum WGS84). Samples were collected in the field and substrate samples were also removed for moist chamber culture. Methods used for collecting myxomycetes in the field and substrates for laboratory culture can be found in Stephenson (1989), and Rossman \& al. (1998).
All the fieldwork was carried out by A. Estrada-Torres, C. Lado and D. Wrigley de Basanta, with the help of S.L. Stephenson, A. Rollins, G. Rebaza and I. Treviño in 2012, with J. Garcia and A. Cano in 2013, and with J. Rojas-Fox, J. Molina and S. Castillo in 2014. Four investigators collected at any one time with approximately one hour spent in each collecting locality.

Moist chamber cultures were prepared using sterile disposable plastic Petri dishes (9 $\mathrm{cm}$ diameter), in the manner described by Wrigley de Basanta \& al. (2009). The pH of each culture was determined with a portable $\mathrm{pH}$ meter after 24 hours, and then excess water in each dish was poured off. Cultures were maintained at room temperature $\left(21-25^{\circ} \mathrm{C}\right)$ in diffuse daylight and examined at regular intervals with a dissecting microscope for a period of up to three months. As the myxomycetes matured, the portion of the substrate upon which they occurred was removed from the moist chamber culture, allowed to dry slowly in a closed empty Petri dish and then glued in a small cardboard box. All sporophores of a given species that developed in the same culture were considered to represent a single record.

The completeness of the sampling effort for the studied region was evaluated using the ACE and the CHAO1 abundance índices (Colwell \& Coddington 1994; Colwell $\&$ al., 2004) and the accumulation curve adjusted according to the Clench function where $S_{n}=(a * n) /[1+(b * n)]$ where $S_{n}$ is the number of species accumulated for a unit of collecting effort (n) (Jimenez-Valverde \& Hortal, 2003). Each collecting site was considered as the unit of collecting effort, using the total number of species found with the program EstimateS v 9.1.0 (Colwell, 2013), adjusted so that 3 was the upper abundance limit for rare or infrequent species, following the criteria of Stephenson \& al. (1993). The latter authors considered species with a relative abundance of $\leq 0.05$ to be rare. With the same program the richness of species with 1.5 times more sampling effort was calculated. The adjustment according to the Clench function was carried out with the program Statistica v 12, using the Simplex and Quasi-Newton method of adjustment (Jimenez-Valverde \& Hortal, 2003). ACE and CHAO1 abundance indices were calculated for all results, only field results and only moist chamber culture results.

To measure the complementarity of the assemblage of myxomycetes in the studied area and other regions in South America, the formula proposed by Colwell \& Coddington (1994) was used: $\mathrm{C}_{\mathrm{jk}}=\mathrm{U}_{\mathrm{jk}} / \mathrm{S}_{\mathrm{jk}}$ where $\mathrm{U}_{\mathrm{jk}}=\mathrm{S}_{\mathrm{j}}+\mathrm{S}_{\mathrm{k}}-\left(2 \mathrm{~V}_{\mathrm{jk}}\right)$ and $S_{j k}=S_{j}+S_{k}-V_{j k}$ where $U_{j k}$ is the number of species that are different for the two communities, $S_{j k}$ is the total richness of species for both communities, $S_{j}$ is the number of species from the first community, $S_{k}$ is the number of species from the second and $V_{j k}$ is the number of species common to both.

To determine whether the proportion of collections and species from the different orders of myxomycete was dependent on the sampling method used, a test of independence was done using a contingency table and a $\mathrm{X}^{2}$ test (Zar, 1976).

All specimens are deposited in the MA-Fungi herbarium (sub Lado) with duplicates in TLXM (sub aet), UARK (sub sls) or in the private collection of Wrigley de Basanta (dwb). 
Table 1. List of collecting localities. [The first name refers to the administrative department and the second to the province. (*) Localities with no collections.]

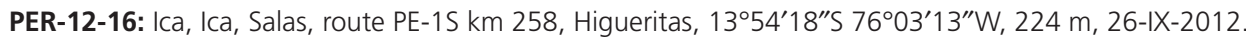

PER-12-17: Ica, Ica, Santiago, route PE-1S km 375, 7 kms W of Santa Cruz, 14²8'59”S 75¹9'57”W, 567 m, 26-IX-2012.

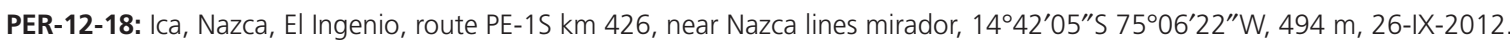

PER-12-19: Ica, Nazca, Nazca, route PE-26A to Puquio km 10.500, 1449'24”S 7451'07”W, 845 m, 26-IX-2012.

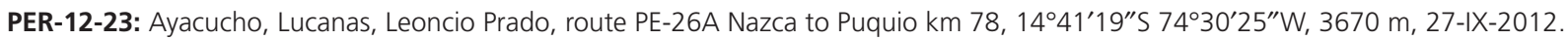

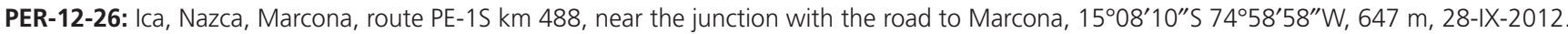

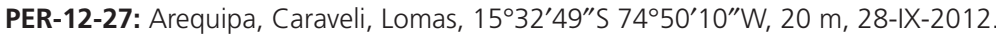

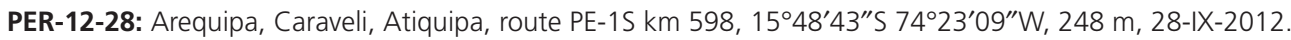

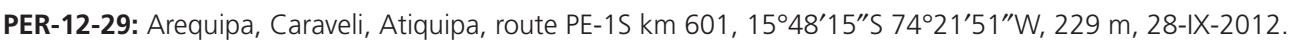

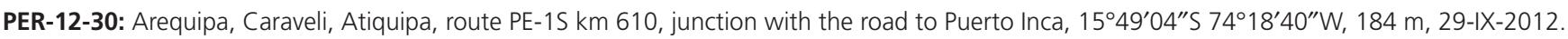

PER-12-31: Arequipa, Caraveli, Atiquipa, route PE-1S km 591, 1546'32"S 74²5’30”W, 240 m, 29-IX-2012.

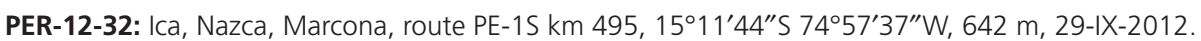

PER-12-33: Ica, Nazca, Marcona, San Juan de Marcona, route PE-30, 11 km SW of the Panamericana junction, $15^{\circ} 12^{\prime} 44^{\prime \prime} \mathrm{S} 75^{\circ} 02^{\prime} 08^{\prime \prime} \mathrm{W}, 748 \mathrm{~m}$, 29-IX-2012.

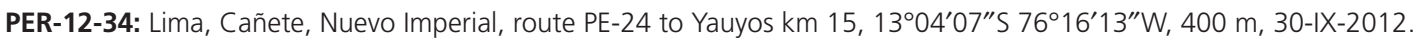

PER-12-35: Lima, Cañete, Zuñiga, arqueological area El Cascajal, route PE-24 km 64, 1250'16"S 7559'26”W, 922 m, $30-I X-2012$

PER-12-39: Lima, Yauyos, Catahuasi, Canchán, route PE-2, km 86, 1246’27"S 7556'04”W, 1300 m, 1-X-2012.

PER-12-40: Lima, Yauyos, Catahuasi, San Jerónimo, route PE-24 km 75, 1249'29”S 7555’00”W, 1060 m, 1-X-2012.

PER-12-41: Arequipa, Islay, Islay, Matarani, route PE-34 km 39, 1654’56"S 7202'50”W, 855 m, 4-X-2012.

PER-12-42: Arequipa, Islay, Islay, Matarani, route PE-34 km 44, 1656'50”S 7203'23”W, 623 m, 4-X-2012.

PER-12-43: Arequipa, Islay, Islay, Matarani, route PE-34 km 46, 1657'26"S 7203'29”W, 548 m, 4-X-2012.

PER-12-47: Tacna, Tacna, Pachia, Miculla, 1752'42"S 7008'43"W, 1175 m, 6-X-2012.

PER-12-48: Tacna, Tacna, Sama, Sama Grande, route PE-1S km 1254, 1748’21"S 70³5'29”W, 515 m, 6-X-2012.

PER-12-49: Tacna, Jorge Basadre, Locumba, route PE-1S km 1233, 1745'49"S 7046’55”W, 690 m, 6-X-2012.

PER-12-50: Tacna, Tacna, Sama, Puerto Grau, Morro Sama, route PE-1SD km 76, 18 km SE of Ite, 1800’02"S 7052'57”W, 53 m, 6-X-2012.

*PER-12-51: Moquegua, Ilo, Ilo, route PE-1SD km 118, Punta Huaca Luna, 1747’05"S 71¹0’32"W, 50 m, 6-X-2012.

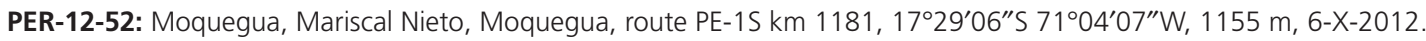

*PER-13-01: Lima, Huaral, Ancón, comunidad Aucallama, route PE-1N km 58, 11²4'16”S 77¹0’39”W, 373 m, 9-IV-2013.

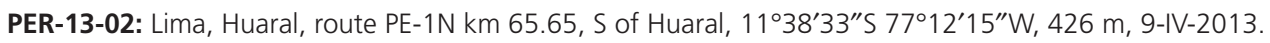

PER-13-03: Lima, Huaral, Santa Rosa, route PE-1NE km 18, E of Lachay, 11॰17’36"S 77¹9’22”W, 350 m, 9-IV-2013.

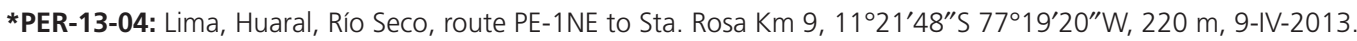

PER-13-05: Lima, Huaura, Sayán, route PE-18 km 59, NE of Sayán, 1103’02"S 7707’34”W, 940 m, 10-IV-2013.

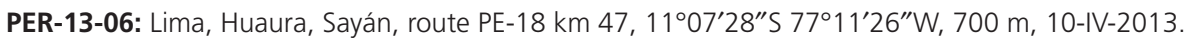

*PER-13-07: Ancash, Huarmey, Gramadal, route PE-1N km 222, Paramonga, 10³5'29"S 7752'12”W, 121 m, $10-\mathrm{IV}-2013$.

*PER-13-08: Ancash, Huarmey, Gramadal, route PE-1N km 237, S of Gramadal, 10²7'54"S 7754’56"W, 198 m, $10-I V-2013$.

*PER-13-09: Ancash, Casma, Casma, route PE-1N km 344, S of Casma, 0943'49"S 78¹5'45”W, 110 m, 10-IV-2013.

PER-13-10: Ancash, Pallasca, Galgada, Tablachaca river, route AN-100, $3.5 \mathrm{~km} N$ of the junction with the Pallasca to Ancos road, $08^{\circ} 28^{\prime} 13^{\prime \prime} \mathrm{S}$ $78^{\circ} 09^{\prime} 20^{\prime \prime} W, 1111 \mathrm{~m}, 11-\mathrm{IV}-2013$

PER-13-12: Ancash, Pallasca, Estación Chuquicara, route PE-3N km 83, 08³3'27"S 78¹3'01"W, 860 m, 11-IV-2013.

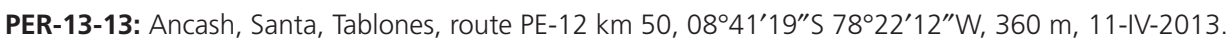

*PER-13-14: La Libertad, Viru, Chaoroute PE-1N km 480, S of Chao, 0842'22"S 78³8'23"W, 305 m, 12-IV-2013.

PER-13-15: La Libertad, Trujillo, Moche, route PE-1N km 549, SE of Moche, 08¹3'40"S 7856'42”W, 247 m, 12-IV-2013.

PER-13-18: La Libertad, Ascope, Paiján, route PE-1N km 638, SE of San Pedro de Lloc, 07³9’08”S 79²0’40”W, 101 m, $13-I V-2013$.

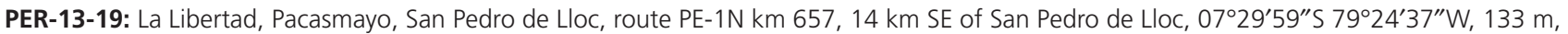
13-IV-2013

PER-13-20: La Libertad, Chepén, Ciudad de Dios, route PE-08 km 18, Mexico, 07018'01"S 79¹9'48”W, 192 m, $13-I V-2013$.

PER-13-21: Cajamarca, Contumaza, Tembladera, route PE-08 Km 60, E of Tembladera, 07¹3'51"S 7903'18”W, 548 m, $13-I V-2013$.

PER-13-27: Cajamarca, San Pablo, Chilete, route PE-08 km 99, 07¹2'53"S 7846’29”W, 967 m, 15-IV-2013.

PER-13-28: Cajamarca, Contumaza, Chilete, route PE-08 km 85.500, 07¹3'37"S 7852'59”W, 816 m, 15-IV-2013.

PER-13-29: La Libertad, Ascope, Chicama, route PE-1N km 592, 0757'15"S 7905'26”W, 217 m, 15-IV-2013. 
Table 1. (Continued).

PER-13-30: Ancash, Casma, Tortugas, route PE-1N km 397.500, 09²0'19"S 78²3'53”W, 165 m, 6-IV-2013.

PER-13-31: Ancash, Casma, Casma, route PE-14 km 22, E of Casma, 09²8'54"S 7807'02"W, 535 m, 17-IV-2013.

PER-13-32: Ancash, Huaraz, Pariacoto, route PE-14 km 62, 09³3’01"S 7750'46”W, 1478 m, 17-IV-2013.

PER-13-36: Ancash, Huaylas, Caraz, Huallanca, Cañón del Pato, route PE-3N km 689, N of Huallanca, $08^{\circ} 48^{\prime} 28^{\prime \prime} S$ 7752’49”W, 1322 m, 18-IV-2013.

PER-13-58: Ancash, Bolognesi, Hornillos, route PE-16 km 58, 10¹4'41"S 77³4'18”W, 1034 m, 24-IV-2013.

*PER-14-01: Piura, Sechura, Parachique, route PE-1NK km 64, to Bayóvar, 546’51"S 8050'21"W, 15 m, 7-V-2014.

PER-14-02: Piura, Sechura, Bayóvar, junction with the road to Chiclayo, route PE-04 km 45, 553'35"S 8054'15”W, 15 m, 7-V-2014.

PER-14-03: Piura, Sechura, Bayóvar, route to Bayóvar km 10, 551'33"S 8100’31”W, 44 m, 7-V-2014.

PER-14-04: Piura, Sechura, route PE-04 km 18, 557'56"S 8040'19”'W, 27 m, 7-V-2014.

PER-14-05: Piura, Sechura, route PE-1N km 926, 548'31"S 80³2'20”W, 18 m, 7-V-2014.

PER-14-06: Piura, Sechura, route PE-1N km 957, 5³1'51"S 80³5'07"W, 20 m, 8-V-2014.

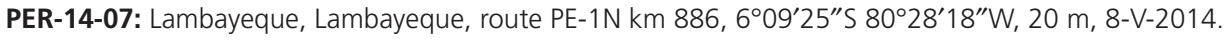

PER-14-08: Lambayeque, Lambayeque, route PE-1N km 870, 6¹5'24"S 80²1'49"W, -15 m, 8-V-2014.

PER-14-09: Lambayeque, Lambayeque, route PE-1N km 830, 6²8'56"S 8005'49”W, 15 m, 8-V-2014.

PER-14-10: Lambayeque, Lambayeque, route PE-1N km 814, Morrope, 6³4'30"S 7959'23"W, 20 m, 8-V-2014.

PER-14-11: La Libertad, route PE-1N km 739, Mocupe, 702'05"S 79³5'27”W, 58 m, 9-V-2014.

PER-14-12: Cajamarca, Chota, route PE-06A km 80, San Carlos, 6³6'49"S 79¹6'35”'W, 343 m, 9-V-2014.

PER-14-13: Lambayeque, Chiclayo, route PE-06A km 66, Chongoyape, La Cascada, 6³7'40"S 79²3'05”W, 250 m, 9-V-2014.

PER-14-14: Lambayeque, Lambayeque, route PE-1NJ km 60, Motupe, Cerro la Vieja, 6¹2'23"S 7942’01"W, 131 m, $10-V-2014$.

*PER-14-15: Lambayeque, Lambayeque, route PE-04E km 14, Olmos, 556'25"S 79³6'22”W, 451 m, 10-V-2014.

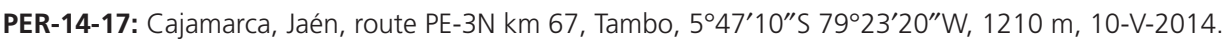

PER-14-18: Cajamarca, Jaén, route PE-02B, 2 km W of Tamborapa, 5²7'40"S 7850'46”'W, 528 m, 11-V-2014.

PER-14-19: Cajamarca, Jaén, route PE-5N km 33, 5³7'39"S 7846’03”W, 750 m, 11-V-2014.

PER-14-20: Cajamarca, Jaén, route PE-5N km 30, 5³8'17"S 7844’59"W, 625 m, 11-V-2014.

*PER-14-21: Cajamarca, Jaén, route PE-04C km 195, 24 de julio bridge, Marañón river, 545'41"S 7841'41”W, 449 m, 11-V-2014.

*PER-14-22: Cajamarca, Cutervo, route PE-3N km 146, 602'36"S 7854'25"W, 720 m, 12-V-2014.

*PER-14-23: Cajamarca, Jaén, route PE-3N km137, Chamaya I bridge, Infiernillo bridge, 601'09"S 7858'27"W, 777 m, $12-V-2014$.

PER-14-24: Cajamarca, Jaén, route PE-3N km 106, Pucará, 6 km SE of the road to Pomahuaca, 558'55”S 79¹1'46”W, 903 m, $12-V-2014$.

PER-14-25: Piura, Huancabamba, route PE-04B km 29, Limón Porculla, 553’41"S 79³1’53”W, 1246 m, 12-V-2014.

PER-14-26: Lambayeque, Lambayeque, route PE-1NJ km 126, Querpón, 543’04"S 7953’27”W, 147 m, 12-V-2014.

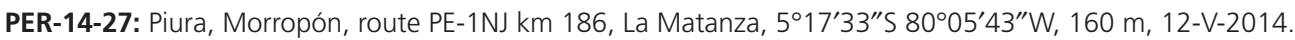

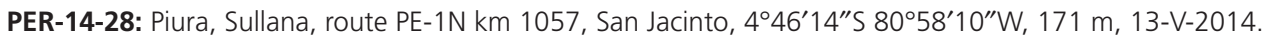

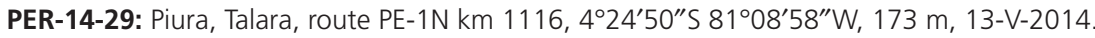

PER-14-30: Tumbes, Contralmirante Villar, route PE-1N km 1220, Palo Santo bridge, 347'14"S 8048'10"W, 4 m, $13-V-2014$.

PER-14-31: Tumbes, Contralmirante Villar, Bocapán, route TU-107 km 7, 344'25"S 8042'49"W, 36 m, 14-V-2014.

PER-14-32: Tumbes, Contralmirante Villar, Trigal, route TU-107 km 20, 349’53"S 8040'44”W, 61 m, 14-V-2014.

PER-14-33: Tumbes, Contralmirante Villar, Avería, route TU-107 km 29, 351'55"S 80³7'43"W, 132 m, 14-V-2014.

PER-14-34: Tumbes, Contralmirante Villar, Pampa el Toro, route TU-108 km 10, 356'53"S 80³3'23"'W, 385 m, 14-V-2014.

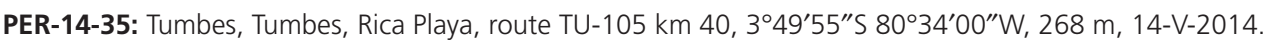

PER-14-36: Tumbes, Tumbes, Vaquería, route TU-105 km13, 341'56"S 80²7'08”W, 29 m, 14-V-2014.

*PER-14-37: Tumbes, Contralmirante Villar, Punta Sal, route PE-1N km 1184, 400’38"S 8058'43"W, 16 m, $15-V-2014$.

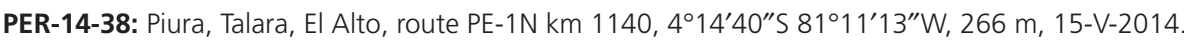

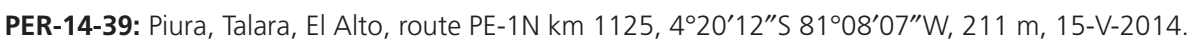

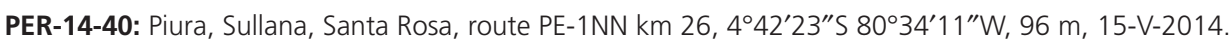

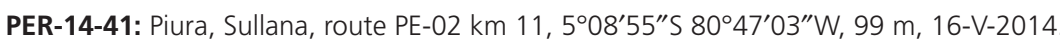

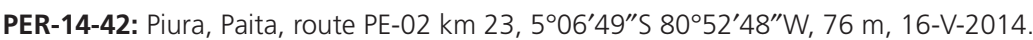

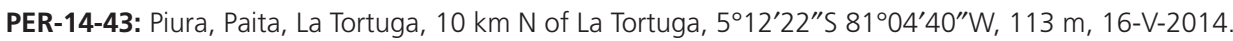

PER-14-47: Lima, Canta, Santa Rosa de Quives, 31 km S of Canta, 11 ${ }^{\circ} 36^{\prime} 49^{\prime \prime} \mathrm{S}$ 7646'16”W, 1390 m, 18-V-2014.

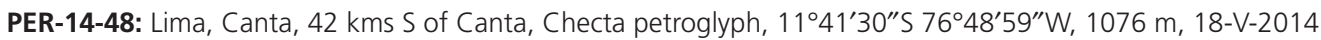




\section{RESULTS}

As a result of the survey carried out in three consecutive years in these hyperarid areas, 723 collections of myxomycetes were identified, either specimens that had developed in the field under natural conditions or those that were recovered from moist chamber cultures. In total, 78 taxa representing 23 genera of myxomycetes were recorded.

\section{Annotated list of species}

Myxomycete collections from this survey are arranged alphabetically in the list that follows by genus and then species. Nomenclature follows Lado (2005-2015) unless otherwise stated. Information is provided on the source of each record (either a field collection or a collection obtained from a moist chamber [mc] culture), the $\mathrm{pH}$ of the culture in which the specimen appeared, the substrate upon which it was collected and the locality from which it was collected (see Table 1). All identified collections are included. Species of particular interest have additional comments. The abbreviation "cf." in the name of a taxon indicates that the specimen representing the source of the record could not be identified with certainty. This sometimes indicates scanty or aberrant material. Comments on the distribution of the species in the Neotropics are based on Lado \& Wrigley de Basanta (2008), but the information on the area of study has been updated with the added references. New records for the Neotropics, South America or Peru are marked with $(\dagger)$, $\left({ }^{\circ}\right)$ or an asterisk $(*)$ respectively.

\section{Arcyria afroalpina Rammeloo}

PER-12-43: twigs, Lado 22075 (MA-Fungi 87860); Heliotropium arborescens twigs, Lado 22094 (MA-Fungi 87888). PER-13-10: Deuterochonia longipetala leaf bases (mc, pH 7.78), dwb 3661 (MA-Fungi 88432); Deuterochonia longipetala leaf bases (mc, pH 7.93), dwb 3666.

This species was described from the mountains of tropical Africa. It is widely distributed in the Neotropics. In Peru it has been previously reported by Rojas \& al. (2011) from Madre de Dios.

\section{Arcyria cinerea (Bull.) Pers.}

PER-12-28: branch of an Asteraceae, Lado 21919 (MA-Fungi 87742); twigs (mc, pH 6.62), sls 28314. PER-12-29: twigs (mc, pH 6.97), sls 28322; ground litter (mc, pH 7.39), sls 30225; ground litter (mc, $\mathrm{pH}$ 7.38), sls 30267. PER-12-33: mixed litter (mc, pH 5.67), sls 31012. PER12-43: twigs, Lado 22069 (MA-Fungi 87853). PER-13-12: Deuterochonid longipetala leaf bases (mc, pH 7.78), dwb 3677. PER-13-32: herb litter, Lado 22652 (MA-Fungi 88028). PER-13-58: twigs (mc, pH 7.2), aet 13068a.

This is probably one of the commonest species in the Neotropics. In Peru only reported from Loreto (Wrigley de Basanta \& al., 2008a), in lowland tropical rainforest. Collection dwb 3677 has been left as cf. since it differs from the others in that the sporocarps were a pale peach colour when fresh, and the capillitium is only ornamented with warts, not spines. All the other characters coincide with this species however, and the spores by SEM are typical.

\section{*Arcyria insignis Kalchbr. \& Cooke}

PER-12-29: Schinus molle wood, Lado 21940 (MA-Fungi 87759). PER-12-34: Tillandsia latifolia inflorescences (mc, pH 5.4), aet 12227 Tillandsia purpurea inflorescences (mc, $\mathrm{pH} 7.2$ ), aet 12230; Tillandsia latifolia leaves, Lado 21959 (MA-Fungi 87778), Lado 21964 (MA-Fungi 87779), Lado 21965 (MA-Fungi 87780), Lado 21967 (MA-Fungi 87782), Lado 21969 (MA-Fungi 87783), Lado 21970 (MA-Fungi 87784), Lado 21971 (MA-Fungi 87785), Lado 21972 (MA-Fungi 87786), Lado 21974 (MA-Fungi 87787); Washingtonia sp. leaves, Lado 21993 (MA-Fungi 87805), Lado 21995 (MA-Fungi 87807); mixed Tillandsia (mc, pH 5.71), sls 28432. PER-12-39: Acacia twigs (mc, pH 6.05), sls 28361. PER-12-42: Alternantera pubiflora twigs and leaves, Lado 22026 (MA-Fungi 87819); Trixis cacalioides twigs, Lado 22047 (MA-Fungi 87835), Lado 22052 (MA-Fungi 87840). PER-12-43: twigs, Lado 22062 (MA-Fungi 87847), Lado 22072 (MA-Fungi 87857), Lado 22074 (MA-Fungi 87859), Lado 22076 (MA-Fungi 87861), Lado 22091 (MA-Fungi 87882). PER-13-58: twigs (mc, pH 7.2), aet 13012 (MA-Fungi 88438). PER-14-25: twigs, Lado 23528 (MA-Fungi 88323).

This species is widely distributed in the Neotropics.

\section{*Arcyria major (G. Lister) Ing}

PER-14-25: twigs, Lado 23527 (MA-Fungi 88322).

In the Neotropics previously reported only from Brazil and Ecuador.

\section{*Badhamia foliicola Lister}

PER-12-42: Alternantera pubiflora twigs and leaves, Lado 22036 (MA-Fungi 87828).

In South America previously reported only from Argentina.

\section{*Badhamia macrocarpa (Ces.) Rostaf.}

PER-13-02: twigs (mc, pH 7.2), aet 13022.

This species is widely distributed in the Neotropics.

\section{* Badhamia melanospora Speg.}

PER-12-16: aerial litter (mc, $\mathrm{pH}$ 5.80), sls 30418; twigs (mc, $\mathrm{pH} 4.39$ ), sls 30593. PER-12-17: twigs (mc, pH 4.57), sls 28849; ground litter (mc, pH 4.81), sls 30090. PER-12-19: aerial twigs (mc, pH 4.76), sls 29176; aerial twigs (mc, pH 4.54), sls 29179; ground litter (mc, pH 4.79), sls 30084; ground litter (mc, pH 4.76), sls 30204; twigs/ground litter (mc, pH 5.05), sls 30218. PER-12-28: ground litter (mc, pH 6.55), sls 28316; aerial litter (mc, pH 5.59), sls 28352; ground litter (mc, pH 6.38), sls 28353; aerial litter (mc, pH 5.04), sls 28853; ground litter (mc, pH 6.70), sls 31400. PER-12-29: aerial litter (mc, pH 5.73), sls 30227. PER-1230: Haageocereus decumbens remains, Lado 21948 (MA-Fungi 87768), Lado 21949 (MA-Fungi 87769), Lado 21950 (MA-Fungi 87770), Lado 21951 (MA-Fungi 87771), Lado 21953 (MA-Fungi 87772), Lado 21954 (MA-Fungi 87773), Lado 21955 (MA-Fungi 87774), Lado 21956 (MA-Fungi 87775), Lado 21957 (MA-Fungi 87776), Lado 21958 (MA-Fungi 87777). PER-12-31: mixed litter (mc, pH 5.87), sls 30368. PER-12-32: aerial/ground litter (mc, pH 5.30), sls 30417. PER-12-34: Cleistocactus sp. remains, Lado 21975 (MA-Fungi 87788), Lado 21976 (MA-Fungi 87789), Lado 21977 (MA-Fungi 87790), Lado 21978 (MA-Fungi 87791), Lado 21979 (MA-Fungi 87792), Lado 21980 (MA-Fungi 87793), Lado 21981 (MA-Fungi 87794), Lado 21982 (MA-Fungi 87795), Lado 21983 (MA-Fungi 87796), Lado 21984 (MA-Fungi 87797), Lado 21985 (MA-Fungi 87798), Lado 21986 (MA-Fungi 87799), Lado 21987 (MA-Fungi 87800), Lado 21988 (MA-Fungi 87801); mixed Tillandsia (mc, pH 4.83), sls 29090. PER12-35: Melocactus peruvianus remains, Lado 21996 (MA-Fungi 87809); Haageocereus acranthus remains, Lado 21997 (MA-Fungi 87810), Lado 21998 (MA-Fungi 87811); Neoraimondia roseiflora remains, Lado 21999 (MA-Fungi 87812). PER-12-40: Cleistocactus decumbens remains, 
Lado 22021 (MA-Fungi 87813). PER-12-41: Corryocactus brachypetalus remains, Lado 22022 (MA-Fungi 87814), Lado 22023 (MA-Fungi 87815). PER-12-48: Haageocereus sp. remains (mc, pH 7.8), aet 12229 , (mc, $\mathrm{pH} 7.4$ ), aet 12270, (mc, $\mathrm{pH} 7.4$ ), aet 12277; Haageocereus sp. remains, Lado 22138 (MA-Fungi 87890). PER-12-50: Neoraimondia aticensis remains, Lado 22140 (MA-Fungi 87891). PER-13-05: cactus remains, Lado 22380 (MA-Fungi 87892); Haageocereus sp. remains, Lado 22381 (MA-Fungi 87893), Lado 22382 (MA-Fungi 87894). PER13-06: cactus remains, Lado 22383 (MA-Fungi 87895). PER-13-10: Melocactus sp. remains, Lado 22384 (MA-Fungi 87896), Lado 22385 (MA-Fungi 87897), Lado 22386 (MA-Fungi 87898); Haageocereus sp. remains, Lado 22387 (MA-Fungi 87899), Lado 22388 (MA-Fungi 87900), Lado 22389 (MA-Fungi 87901); Neoraimondia sp. remains, Lado 22390 (MA-Fungi 87902), Lado 22391 (MA-Fungi 87903), Lado 22392 (MA-Fungi 87904). PER-13-12: Haageocereus sp. remains, Lado 22411 (MA-Fungi 87909), Lado 22412 (MA-Fungi 87910), Lado 22413 (MA-Fungi 87911), Lado 22414 (MA-Fungi 87912), Lado 22415 (MA-Fungi 87913), Lado 22416 (MA-Fungi 87914), Lado 22418 (MA-Fungi 87916). PER-13-13: Neoraimondia sp. remains, Lado 22419 (MA-Fungi 87917). PER-13-20: Haageocereus sp. remains, Lado 22523 (MA-Fungi 87951), Lado 22524 (MA-Fungi 87952); Neoraimondia sp. remains, Lado 22527 (MA-Fungi 87955), Lado 22530 (MA-Fungi 87958), Lado 22532 (MA-Fungi 87961), Lado 22533 (MA-Fungi 87962). PER-13-21: Neoraimondia sp. remains, Lado 22534 (MA-Fungi 87963), Lado 22535 (MA-Fungi 87964), Lado 22536 (MA-Fungi 87965), Lado 22537 (MA-Fungi 87966), Lado 22538 (MA-Fungi 87967); Haageocereus sp. remains, Lado 22539 (MA-Fungi 87968), Lado 22540 (MA-Fungi 87969), Lado 22541 (MA-Fungi 87970), Lado 22542 (MA-Fungi 87971) PER-13-28: Espostoa sp. remains, Lado 22612 (MA-Fungi 87991). PER-13-31: Haageocereus limensis remains, Lado 22635 (MA-Fungi 88012), Lado 22636 (MA-Fungi 88013); Armatocereus sp. remains, Lado 22637 (MA-Fungi 88014), Lado 22638 (MA-Fungi 88015), Lado 22639 (MA-Fungi 88016). PER-13-36: Melocactus sp. remains, Lado 22750 (MA-Fungi 88041), Lado 22751 (MA-Fungi 88042), Lado 22752 (MA-Fungi 88043); Haageocereus sp. remains, Lado 22753 (MA-Fungi 88044); Espostoa sp. remains, Lado 22754 (MA-Fungi 88045); Melocactus sp. remains, Lado 22756 (MA-Fungi 88047). PER13-58: Armatocereus procerus remains, Lado 23182 (MA-Fungi 88048); Melocactus peruvianus remains, Lado 23183 (MA-Fungi 88049). PER14-12: Neoraimondia sp. remains, Lado 23426 (MA-Fungi 88090). PER-14-13: Melocactus sp. remains, Lado 23435 (MA-Fungi 88281), Lado 23436 (MA-Fungi 88282), Lado 23437 (MA-Fungi 88283), Lado 23438 (MA-Fungi 88284), Lado 23439 (MA-Fungi 88285), Lado 23440 (MA-Fungi 88286); Espostoa sp. remains, Lado 23441 (MA-Fungi 88287); Haageocereus sp. remains, Lado 23442 (MA-Fungi 88288). PER 14-14: Haageocereus sp. remains, Lado 23443 (MA-Fungi 88289), Lado 23444 (MA-Fungi 88290), Lado 23445 (MA-Fungi 88291), Lado 23446 (MA-Fungi 88292). PER-14-17: Armatocereus sp. remains, Lado 23502 (MA-Fungi 88296). PER-14-30: cactus remains, Lado 23548 (MA-Fungi 88337). PER-14-31: Armatocereus sp. remains, Lado 23549 (MA-Fungi 88338). PER-14-32: Armatocereus sp. remains, Lado 23551 (MA-Fungi 88340), Lado 23552 (MA-Fungi 88341). PER-14-33: Armatocereus sp. remains, Lado 23554 (MA-Fungi 88342), Lado 23555 (MA-Fungi 88343), Lado 23556 (MA-Fungi 88344), Lado 23557 (MA-Fungi 88345). PER-14-34: Armatocereus sp. remains, Lado 23561 (MA-Fungi 88349). PER-14-35: Armatocereus sp. remains, Lado 23564 (MA-Fungi 88352). PER-14-36: Armatocereus sp. remains, Lado 23565 (MA-Fungi 88353), Lado 23566 (MA-Fungi 88354), Lado 23567 (MA-Fungi 88355). PER14-40: Armatocereus sp. remains, Lado 23575 (MA-Fungi 88363), Lado 23576 (MA-Fungi 88364), Lado 23577 (MA-Fungi 88365), Lado 23578 (MA-Fungi 88366). PER-14-47: Haageocereus sp. remains, Lado 23648 (MA-Fungi 88417), Lado 23649 (MA-Fungi 88418). PER-14-48: Haageocereus sp. remains, Lado 23650 (MA-Fungi 88419), Lado 23651 (MA-Fungi 88420).

This species is widely distributed throughout the Neotropics. It is one of the most frequently collected species, 139 collections, especially on cacti and succulent plants, many of which are endemic to the study area.

Ceratiomyxa fruticulosa (O.F. Müll.) T. Macbr.

PER-14-20: cactus remains, Lado 23522 (MA-Fungi 88317).
A very common species in the Neotropics but in Peru previously reported only from the Amazon basin (Stephenson \& Mitchell, 1994; Rojas \& al., 2008; Wrigley de Basanta \& al., 2008a).

\section{${ }^{\circ}$ Comatricha ellae Härk.}

PER-14-06: Prosopis sp. bark (mc, pH 4.81), dwb 3695.

This is a small collection of very typical sporocarps. Originally described on bark from Norway, this is the first record of the species for South America. In the Neotropics it has been previously reported from Mexico.

\section{Comatricha laxa Rostaf.}

PER-12-26: ground litter (mc, pH 4.47), sls 28365 (MA-Fungi 88444); ground litter (mc, pH 4.59), sls 28367. PER-12-34: mixed Tillandsia (mc, $\mathrm{pH}$ 5.71), sls 28360; mixed Tillandsia (mc, pH 4.83), sls 28368. PER12-43: twigs, Lado 22060 (MA-Fungi 87845). PER-12-47: Tillandsia purpurea leaf bases (mc, pH 7.43), dwb 3557. PER-12-49: Ephedra americana twigs (mc, $\mathrm{pH}$ 5.1), aet 12252. PER-13-10: Deuterochonia longipetala leaf bases (mc, pH 7.66), dwb 3646. PER-13-58: twigs (mc, $\mathrm{pH}$ 7.2), aet 13013

In Peru previously reported by Rojas \& al. (2011) from Madre de Dios. Otherwise reported from many countries in the Neotropics. Collections sls 28365, 28367, 28360 and 28368 were characterized by a more globose sporotheca and a more reduced capillitium than those typical of this species, but otherwise they fit the published descriptions of Comatricha laxa.

\section{Comatricha pulchella (C. Bab.) Rostaf.}

PER-13-58: twigs (mc, pH 7.2), aet 13068b.

In Peru previously reported by Rojas \& al. (2011) from Madre de Dios.

Craterium leucocephalum (Pers. ex J.F. Gmel.) Ditmar

PER-13-32: herb litter, Lado 22646 (MA-Fungi 88022). PER-14-24: Capparis scabrida leaves, Lado 23524 (MA-Fungi 88319), Lado 23525 (MA-Fungi 88320).

A widely distributed species in South America, but in Peru previously reported only by Rojas \& al. (2011) from Madre de Dios. The collections Lado 23524 and Lado 23525 are the var. scyphoides not previously reported from the country.

\section{Cribraria violacea Rex}

PER-14-19: Agave americana leaves, Lado 23515 (MA-Fungi 88310).

A widely distributed species in the Neotropics but in Peru previously reported only by Rojas \& al. (2011) in Madre de Dios.

\section{*Dictydiaethalium plumbeum (Schumach.) Rostaf.}

PER-13-27: twigs and litter under Schinus molle, Lado 22606 (MA-Fungi 87984).

This species is widely distributed throughout the Neotropics. 


\section{${ }^{\circ}$ Diderma acanthosporum Estrada \& Lado}

PER-13-32: wood, Lado 22640 (MA-Fungi 88017).

In the Neotropics the species is only known from Mexico. This collection has groups of 3-8 sporocarps. They have dark brown spores 17-20 $\mu \mathrm{m}$ diam, that are paler on one side and ornamented with the characteristic prominent, sharp spines of 2-3 $\mu \mathrm{m}$ in length.

\section{Diderma effusum (Schwein.) Morgan}

PER-14-18: leaf litter, Lado 23507 (MA-Fungi 88301).

Widely distributed throughout the Neotropics. In Peru previously reported only from Madre de Dios (Rojas \& al., 2011).

\section{Diderma hemisphaericum (Bull.) Hornem.}

PER-13-27: twigs and litter under Schinus molle, Lado 22607 (MA-Fungi 87985). PER-13-32: herb litter, Lado 22647 (MA-Fungi 88023), Lado 22648 (MA-Fungi 88024), Lado 22653 (MA-Fungi 88029), Lado 22654 (MA-Fungi 88030).

In Peru previously reported only from Loreto (Wrigley de Basanta \& al., 2008a).

\section{Didymium anellus Morgan}

PER-12-43: twigs, Lado 22092 (MA-Fungi 87883), Lado 22068 (MA-Fungi 87852), Lado 22070 (MA-Fungi 87854). PER-13-20: Neoraimondia sp. remains, Lado 22526 (MA-Fungi 87954).

This species is widely distributed throughout the Neotropics, but not common. In Peru previously reported from Lima and Tumbes (Clark \& Landolt, 2001).

\section{*Didymium anomalum (Rostaf.) Massee}

PER-12-52: Tillandsia purpurea inflorescences (mc, pH 6.9), aet 12256.

The Peruvian collection fits the description of the species given by Poulain \& al. (2011) except that in the plasmodiocarps no columella is evident. In the sporocarpic forms however a pulvinate calcareous base is present. The spores are 12.6-13.4 $\mu \mathrm{m}$ diam and the capillitium is typical for the species, hyaline and forming a complete net. In South America it has only been recorded previously from Argentina (Moreno \& al., 2012).

\section{*Didymium applanatum Nann.-Bremek.}

PER-12-32: Tillandsia sp. inflorescence (mc, pH 6.9), aet 12232, (mc, pH 7.3), aet $12235 \mathrm{a},(\mathrm{mc}, \mathrm{pH} 5.6$ ), aet 12257 , (mc, pH 6.9), aet 12283. PER-12 33: Tillandsia purpurea inflorescence (mc, $\mathrm{pH} 7.2)$, aet 12278, (mc, $\mathrm{pH} 7.0$ ), aet 12279. PER-12-34: Tillandsia purpurea inflorescence (mc, $\mathrm{pH} 7.3$ ), aet 12226, (mc, pH 7.0), aet 12228, (mc, pH 7.3), aet 12251. PER-13-10: Puya sp. inflorescence (mc, pH 5.3), aet 13095.

Collections aet 12226, 12228, 12232, 12235a, 12279 and 13095 have flattened sporothecae with a non-calcareous base, no columella, a hyaline peridium and spores that measure 7-7-11.3 $\mu \mathrm{m}$, with groups of warts. These characters agree with those noted by Poulain \& al. (2011) for Didymium applanatum except for the stalks, which are variable from calcareous to partially or completely without lime and dark in colour, filled with refuse matter and so looking macroscopically like Didymium clavus. Collections aet 12251, 12258 and 12278 have smaller hemispherical sporothecae, but the bases are not umbilicate and they have no columella. Their spore size overlaps with the previous samples, the capilitium is similar and the stalks are bi-coloured with a calcareous apex and darker base, like some of those above. In collection aet 12283 both forms were mixed so it was considered that they probably represent extremes of the same species. Didymium applanatum has recently been considered to be a synonym of $D$. squamulosum var. claviforme Sturgis (Oltra, 2003), a very variable species. However given that the characters of the Peruvian collections are fairly constant with flattened sporothecae, non umbilicate bases and without a columella we prefer to keep the species separate for the moment until further evidence like molecular data combine or separate these forms.

In the Neotropics it was only previously recorded in Mexico and Ecuador.

\section{*Didymium bahiense Gottsb.}

PER-13-32: Furcraea occidentalis leaves, Lado 22656 (MA-Fungi 88032).

Originally described from Brazil (Gottsberger, 1968), it is widely distributed throughout the Neotropics.

Didymium clavus (Alb. \& Schwein.) Rabenh.

PER-12-26: aerial litter (mc, pH 5.60), sls 30118.

A common species in the Neotropics, but in Peru previously reported only from Madre de Dios (Rojas \& al., 2011).

\section{*Didymium columellacavum Hochg., Gottsb. \&} Nann.-Bremek. Figs. 17-19.

PER-12-29: twigs and leaves of an Asteraceae, Lado 21933 (MA-Fungi 87750), Lado 21935 (MA-Fungi 87752), Lado 21937 (MA-Fungi 87755) Lado 21938 (MA-Fungi 87757); leaves of an Asteraceae, Lado 21944 (MA-Fungi 87763); twigs and leaves of an Asteraceae, Lado 21946 (MA-Fungi 87765). PER-13-32: herb litter, Lado 22649 (MA-Fungi 88025), Lado 22651 (MA-Fungi 88027).

This species has only been reported previously from Brazil. These specimens show the typical hollow clubshaped columella, (Figs. 17-18), filled with lime crystals. The stalk is darker and filled with refuse matter below, and the external surface covered with lime crystals, that are less abundant towards the base. The spores by SEM are densely warted and some warts are fused in a short subreticulate pattern (Fig. 19).

\section{Didymium difforme (Pers.) Gray}

PER-12-33: Tillandsia sp. litter (mc, pH 6.95), dwb 3537. PER-12-34 Tillandsia purpurea inflorescence (mc, $\mathrm{pH} 7.3$ ), aet 12231b. PER-12-35: Haageocereus acranthus flowers (mc, $\mathrm{pH} 7.0$ ), aet (a) 12239. PER-12-47: Tillandsia purpurea inflorescence (mc, pH 6.0), aet 12281. PER-12-52: Tillandsia purpurea inflorescence (mc, $\mathrm{pH}$ 6.7), aet 12331, (mc, pH 6.9), aet 12348. PER-13-32: ground litter (mc, pH 6.53), sls 31104.

Widely distributed throughout the Neotropics. In Peru previously reported only from Madre de Dios (Rojas \& al., 2011). Specimen aet 12239 , isolated from cactus flowers, has large flat branched or ring-shaped plasmodiocarps with an egg-shell like outer layer that is yellowish. Microscopically 
it has the typical characters of $D$. difforme, spores 11.8-13.0 $\mu \mathrm{m}$, with a paler area. It is possibly an ecotype of the species but has been left as cf.

\section{*Didymium dubium Rostaf. Fig. 38.}

PER-12-43: Heliotropium arborescens twigs, Lado 22093 (MA-Fungi 87884). PER-12-52: Tillandsia purpurea (mc, $\mathrm{pH}$ 6.7), aet 12262. PER-13. 21: twigs, Lado 22554 (MA-Fungi 87982).

Widely distributed throughout the Neotropics. The spores of the collection Lado 22554 are illustrated in the Fig. 38, and are densely warted.

\section{$\lceil$ Didymium nigrisporum Nann.-Bremek., K.G. Mukerji \& Pasricha Figs. 22-24.}

PER-12-16: ground litter (mc, pH 6.50), sls 30008. PER-12-17: Tillandsio purpurea leaf bases (mc, pH 6.01), dwb 3555 (MA-Fungi 88423); ground litter (mc, pH 4.81), sls 30184; aerial litter (mc, pH 5.52), sls 30186; ground litter (mc, pH 4.79), sls 30189; aerial litter (mc, pH 4.78), sls 30272. PER-12-18: twigs/ground litter (mc, pH 4.50), sls 30187. PER-1219: twigs/ground (mc, pH 5.05), sls 30174. PER-12-26: ground litter (mc, pH 4.50), sls 28427. PER-12-28: branch of an Asteraceae, Lado 21918 (MA-Fungi 87741); ground litter (mc, pH 6.70), sls 28312; aerial litter (mc, pH 5.59), sls 28854. PER-12-29: twigs and leaves of an Asteraceae, Lado 21932 (MA-Fungi 87749), Lado 21935 (MA-Fungi 87753); leaves of an Asteraceae, Lado 21945 (MA-Fungi 87764); aerial litter (mc, pH 5.73), sls 30075; aerial litter (mc, pH 5.84), sls 30091; aerial litter (mc, $\mathrm{pH}$ 6.13), sls 30108; ground litter (mc, pH 5.28), sls 30109. PER-12-31: mixed litter (mc, $\mathrm{pH}$ 4.65), sls 30117. PER-12-32: aerial and ground litter (mc, pH 5.25), sls 30009. PER-12-34: Tillandsia purpurea inflorescence (mc, $\mathrm{pH} 7.3$ ), aet 12307; mixed Tillandsia (mc, pH 4.83), sls 28320 (MA-Fungi 88441). PER-12-35: Haageocereus acranthus flowers (mc, $\mathrm{pH}$ 8.1), aet 12237. PER-12-42: Alternantera pubiflora twigs and leaves, Lado 22025 (MA-Fungi 87818), Lado 22028 (MA-Fungi 87821), Lado 22029 (MA-Fungi 87822), Lado 22037 (MA-Fungi 87829); Trixis cacalioides twigs, Lado 22038 (MA-Fungi 87830), Lado 22039 (MA-Fungi 87831), Lado 22042 (MA-Fungi 87833), Lado 22046 (MA-Fungi 87834), Lado 22054 (MA-Fungi 87841), Lado 22032 (MA-Fungi 87825), Lado 22034 (MA-Fungi 87826); Trixis cacalioides twigs, Lado 22040 (MA-Fungi 87832), Lado 22048 (MA-Fungi 87836). PER-12-43: twigs, Lado 22057 (MA-Fungi 87842), Lado 22064 (MA-Fungi 87849), Lado 22070 (MA-Fungi 87855), Lado 22078 (MA-Fungi 87863), Lado 22083 (MA-Fungi 87871), Lado 22081 (MA-Fungi 87867), Lado 22087 (MA-Fungi 87878), Lado 22088 (MA-Fungi 87879); Heliotropium arborescens twigs, Lado 22093 (MA-Fungi 87885). PER-12-47: Tillandsia purpurea inflorescence (mc, $\mathrm{pH}$ 6.8), aet 12266; (mc, $\mathrm{pH}$ 6.6), aet 12332. PER-12-52: Tillandsia purpurea litter (mc, $\mathrm{pH}$ 6.41), dwb 3569. PER-13-02: Twigs (mc, pH 7.2), aet 13023, (mc, pH 6.9), aet 13026 (MA-Fungi 88440). PER-13-03: Tillandsia latiflora inflorescence (mc, $\mathrm{pH}$ 6.0), aet 13015 (MA-Fungi 88439), (mc, pH 6.3), aet 13016. PER13-32: herb litter, Lado 22650 (MA-Fungi 88026). PER-14-14: twigs, Lado 23447 (MA-Fungi 88293); leaf litter, Lado 23448 (MA-Fungi 88294), Lado 23449 (MA-Fungi 88295). PER-14-27: Capparis scabrida leaves, Lado 23529 (MA-Fungi 88324), Lado 23530 (MA-Fungi 88325); twigs and leaves, Lado 23537 (MA-Fungi 88327). PER-14-28: Capparis ovalifolia fruit, Lado 23538 (MA-Fungi 88328). PER-14-29: twigs and leaves of Capparis scabrida, Lado 23546 (MA-Fungi 88335), Lado 23547 (MA-Fungi 88336). PER-14-31: Armatocereus sp. remains, Lado 23550 (MA-Fungi 88339). PER-14-43: Capparis scabrida leaves, Lado 23615 (MA-Fungi 88410).

This is the second record of this species in the world. Previously only known from India (NannengaBremekamp \& al., 1984), but surprisingly one of the most frequently found species in this study area, 65 collections. This species is close to $D$. squamulosum and D. muscorum Lakhanpal and Mukerji, but it can be distinguised from both by its very dark, densely warted and usually angular or polygonal spores (Figs. 22-24). The Peruvian specimens agree, in general, with the description of the original material from India (Nannenga-Bremekamp $\&$ al., 1984). The spores however are 12-15(-16) $\mu \mathrm{m}$ diam, a value higher than that reported for the Indian material (11.5-12.5 $\mu \mathrm{m}$ diam). Notable variability was also observed in some of the characters of the species, such as the density of the lime of the peridium. In some cases lime was scanty but in others forming scales or even crusts. The length of the stalk, the shape of the columella, from discoid to subglobose, and the shape of the sporotheca, umbillicate below but not in all the sporocarps were other variable characters. The thickened areolae of the peridium, mentioned in the original description, is only evident in specimens Lado 22083 and Lado 22025, in the rest the colour is uniform.

\section{Didymium ochroideum G. Lister}

PER-12-28: aerial litter (mc, pH 5.59), sls 28351 (MA-Fungi 88442). PER13-21: leaf litter, Lado 22543 (MA-Fungi 87972).

In Peru previously reported from Madre de Dios (Rojas $\&$ al., 2011). Collection Lado 22543 has sessile sporocarps or short plasmodiocarps with the typical aereolate peridium that is characteristic of the species. The colour of the sporocarps however is whitish and the spores are larger than the dimensions in the literature, $10-12 \mu \mathrm{m}$ diam vs 6-7(-8) $\mu \mathrm{m}$ (Martin \& alexopoulos, 1969). For this reason the specimen has been left as cf.

\section{Didymium peruvianum Lado, D. Wrigley \& S.L. Stephenson, sp. nov. Figs. 25-37.}

\section{Mycobank: MB 815696.}

Sporophores sporocarpic, dispersed or grouped to sometimes gregarious, whitish, flattened, diminutive. Sporocarps sessile or with a narrow base to subsessile, discoid, (0.15-) 0.25-0.5 (-0.6) mm diam., 0.064-0.160 mm high, sometimes slightly umbilicate above (Figs. 25-26). Sporotheca covered with a continuous layer of calcium carbonate crystals, egg-shell like (Fig. 26), yellowish gray (93. y Gray) to light gray (264. 1. Gray) or almost white (263. White), the base darker, light yellowish brown (79. 1. gy. y $\mathrm{Br}$ ) to grayish brown (61. gy. Br). Hypothallus inconspicuous. Stalk very short, 0.02-0.05 mm tall, often represented by only a restricted base of the sporotheca, brownish. Peridium double, the layers appressed, the outer layer calcareous, consisting of a smooth lime-shell of small, densely packed, lime crystals; the inner layer membranous and colourless, with a smooth inner surface, also by SEM, showing the impression of the spores (Figs. 32-35); dehiscence circumscissile, by a well-defined lid, after which a small peridial rim remains around the base of the sporotheca (Fig. 26). Columella absent. Capillitium scanty, filiform (Fig. 27), threads 1-2 $\mu \mathrm{m}$ diam, scarcely branched, with few cross connections, brown $(58 . \mathrm{m}$. Br-77. m. y Br), smooth. Spores free, black in mass, brown (59. d. Br-62. d. gy. Br) with broad pale bands (57. 1. Br-60. 1. gy. Br) by TL (Figs. 28-31), subglobose, (11.5-) 12.5-14 (-15) $\mu \mathrm{m}$ diam, warted, by LM the dark areas appear to have more strongly pigmented and thus more prominent warts, uniformly warted by SEM (Figs. 36-37). Plasmodium not observed. 

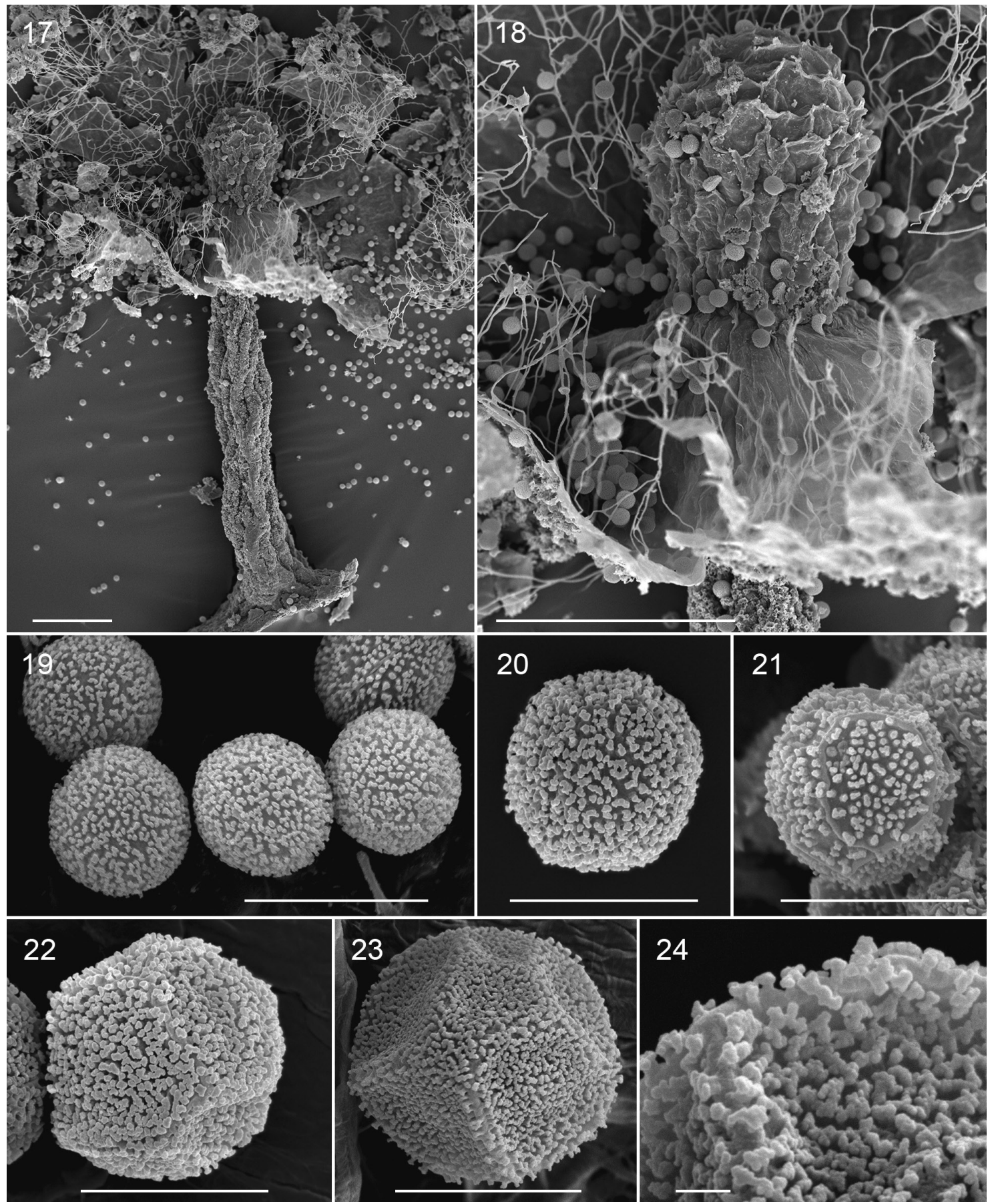

Figs. 17-24. Didymium columellacavum (Lado 22649). 17. Sporocarp showing the columella, the capillitium, the peridium and the stalk with the external surface covered with lime crystals. 18. Detail of the club-shaped columella. 19. Spores. 20-21. Didymium squamulosum. Fig. 20. Spore (Lado 22547). 21. Spores (Lado 23526). 22-24. Didymium nigrisporum. 22. Polygonal spore densely warted (Lado 21945). 23. Polygonal spore densely warted (Lado 23537). 24. Detail of the ornamentation of the spore (Lado 23537). Bar: 17-18=100 $\mu \mathrm{m} ; 19-23=10 \mu \mathrm{m} ; 24=1 \mu \mathrm{m}$.

HOLOTYPE. PERU. Ayacucho: Lucanas, Leoncio Prado, route PE-26A Nazca to Puquio km 78, 1441'19"S $74^{\circ} 30^{\prime} 25^{\prime \prime} \mathrm{W}, 3670 \mathrm{~m}, 27-\mathrm{IX}-2012$, (PER-12-23), aerial litter (mc, pH 5.23), sls 28943 (MA-Fungi 88473).

Other specimens examined. PERU. Ayacucho: Lucanas, Leoncio Prado, route PE-26A Nazca to Puquio

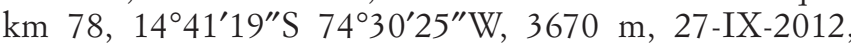
(PER-12-23), aerial litter (mc, pH 4.44), sls 28941 (MA-Fungi 88474). Arequipa: Caraveli, Atiquipa, route

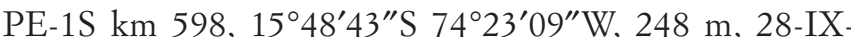
2012, (PER-12-28), ground litter (mc, pH 6.38), sls 28355 (MA-Fungi 88475). Arequipa: Caraveli, Atiquipa, route

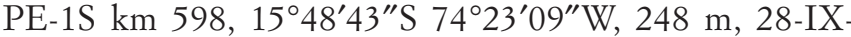
2012, twigs of an Asteraceae, Lado 21925 (MA-Fungi 88472).

Etymology. The epithet peruvianum refers to the geographic area where the species was first found.

Habitat. On dead twigs, aerial and ground plant litter. 
Distribution. Known only from the South and Central Peru (Ayacucho and Arequipa regions), from sea level to almost $4000 \mathrm{~m}$.

The principal characters that distinguish this minute species are the brown spores with broad pale bands (Figs. 28-31) and groups of more prominent pigmented warts, and the eggshell-like outer peridium (Figs. 25-26). Other Didymium species covered with an eggshell-like layer of packed lime crystals include D. annulisporum H.W. Keller \& Schokn. D. trachysporum G. Lister, D. listeri Massee, D. rugulosporum Kowalski, D. quitense (Pat.) Torrend and D. difforme (Pers.) Gray. The first three have smaller spores from 8-10 um diam. vs $12.5-14 \mu \mathrm{m}$ in the new species (Figs. 28-31,36). Didymium rugulosporum has larger spores $(18-20 \mu \mathrm{m})$ and a dense rigid capillitium forming a wide meshed reticulum whereas D. peruvianum has scanty capillitium, scarcely branched and with few cross connections (Fig. 27) Didymium quitense has very different spores with large warts forming a network of irregular muri by SEM (Lado \& al., 2011). Didymium difforme also has different spores, pale on one side and densely covered with very fine warts (Nannenga-Bremekamp, 1975; Neubert \& al., 1995: 340). Didymium nullifilum (Kowalski) M.L. Farr has densely compacted lime scales on the peridium as well as smaller spores $(8-10 \mu \mathrm{m}$ diam. vs $12.5-14 \mu \mathrm{m})$ and the spores have scattered prominent spines (Kowalski, 1972). Short stalked species that could be confused with $D$. peruvianum include D. umbilicatum, D. mexicanum and D. nigrisporum but they all have calcareous stalks not very short and brownish stalks or merely a restricted base to the sporotheca (Wrigley de Basanta \& al., 2008b; Moreno \& al., 1997; Nannenga-Bremekamp \& al., 1984). The first two are also umbilicate above, but both have a single membranous peridium and scattered lime crystals, and are not covered with a continuous layer of eggshell like lime. They also have different spores. Didymium nigrisporum Nann.-Bremek., K.G. Mukerji \& Pasricha also has very dark, strongly warted spores and not broad pale bands between the more prominent warts. The colour of the sporocarps in D. inconspicuum Nann.-Bremek. \& D.W. Mitch. and D. saturnus H.W. Keller differentiate these from the new species since the former is ochraceous to fawn (Nannenga-Bremekamp, 1989) and the latter straw yellow to silvery gray or brown (Keller, 1970). In addition both have abundant capillitium and D. saturnus has spores with a prominent ring. Some other small, sessile Didymium species are D. comatum (Lister) Nann.-Bremek., D. circumscissile K.D. Whitney \& L.S. Olive and D. atrichum Henney \& alexop. In D. comatum the capillitium is profuse, expanding elastically when the lid comes off, not scanty like in D. peruvianum and the spores are violaceous, not brown, and paler on one side, minutely warted, and the warts are arranged in rows, sometimes forming a small-meshed reticulum (Nannenga-Bremekamp, 1966). The shape of the sporocarps in $D$. circumscissile is different from the new species as they are globose, obovate or turbinate, occasionally reniform, not discoid. The distribution of lime is also different as the sporotheca is lightly sprinkled with crystals, especially on the operculum (Whitney and Olive, 1983), whereas in $D$. peruvianum it is a layer like an eggshell. In D. atrichum capillitium is totally lacking and it has smaller spores that have spinulose ornamentation arranged in a reticulate lacey pattern (Henney \& al., 1980), not with broad pale bands between more prominent warts.
Didymium peruvianum was collected in the field, where it fruited under natural conditions, and was also isolated from plant litter put into moist chamber culture. The collections are from two different regions in Peru and at very different elevations. The combination of characters do not match any described species of Didymium and so we describe it here as a new species.

\section{*Didymium quitense (Pat.) Torrend}

PER-14-27: Capparis scabrida leaves, Lado 23533 (MA-Fungi 88326). PER14-39: Capparis ovalifolia leaves, Lado 23571 (MA-Fungi 88359).

This species, described originally from Quito (Ecuador) as Chondrioderma quitense Pat. (Patouillard \& Lagerheim, 1895) has also been reported from Brazil (Farr, 1968), Argentina (Lado \& al., 2011, 2014) and Chile (Lado \& al., 2013). This is the first record for Peru.

\section{$\dagger$ Didymium cf. rugulosporum Kowalski}

PER-12-47: Tillandsia purpurea inflorescence (mc, pH 6.8), aet 12264.

This is a new record for the Neotropics. The Peruvian material is macroscopically similar to $D$. difforme but it has spores with prominent warts sometimes connected in lines and a wide mesh reticulum. This is a character of D. rugulosporum, a species described by Kowalski (1969) from the United States and known so far only from the type locality. The spores of the Peruvian material however measure 11.8-15.0 $\mu \mathrm{m}$ diam, while Kowalski describes the US material as having spores of $18-22 \mu \mathrm{m}$ diam, and so the collection has been left as cf.

\section{Didymium squamulosum (Alb. \& Schwein.) Fr. Figs. 20-21.}

PER-12-43: twigs, Lado 22082 (MA-Fungi 87869), Lado 22085 (MA-Fungi 87875), Lado 22086 (MA-Fungi 87877), Lado 22089 (MA-Fungi 87880). PER-13-21: leaf litter, Lado 22544 (MA-Fungi 87973); wood, Lado 22547 (MA-Fungi 87976); twigs, Lado 22549 (MA-Fungi 87978), Lado 22552 (MA-Fungi 87981). PER-13-32: ground litter (mc, pH 6.53), sls 31143. PER-14-25: living twigs and leaves, Lado 23526 (MA-Fungi 88321).

Widely distributed throughout the Neotropics. In Peru previously reported from Loreto (Wrigley de Basanta \& al., 2008a). The spores of the collection Lado 22547 are illustrated by SEM (Figs. 20-21) and are typical in form (globose) and size (10-11 $\mu \mathrm{m}$ diam). The collection Lado 23526 has spores that fit the dimensions of the species, 10-11 $\mu \mathrm{m}$ diam, but are densely warted and slightly angular when observed by SEM (Fig. 21). This ornamentation, has also been reported by Nannenga-Bremekamp (1991) for specimens from temperate zones, and could be an ecotype of this variable species. The delimitation of some of these collections with $D$. nigrisporum was difficult but the dimension of the spores 10-11(-12) $\mu \mathrm{m}$ diam vs $12-15(-16) \mu \mathrm{m}$ diam in D. nigrisporum (Figs. 22-23) was the main character used to separate them.

\section{Didymium vaccinum (Durieu \& Mont.) Buchet}

PER-12-27: Unidentified Asteraceae wood (mc, pH 5.6), aet 12358 PER-12-33: Tillandsia sp. inflorescence (mc, $\mathrm{pH}$ 7.2), aet 12347. PER13-10: Deuterochonia longipetala leaf bases (mc, $\mathrm{pH} 7.93$ ), dwb 3664; Deuterochonia longipetala leaf bases (mc, $\mathrm{pH} 7.78$ ), dwb 3672. PER-13-12: 

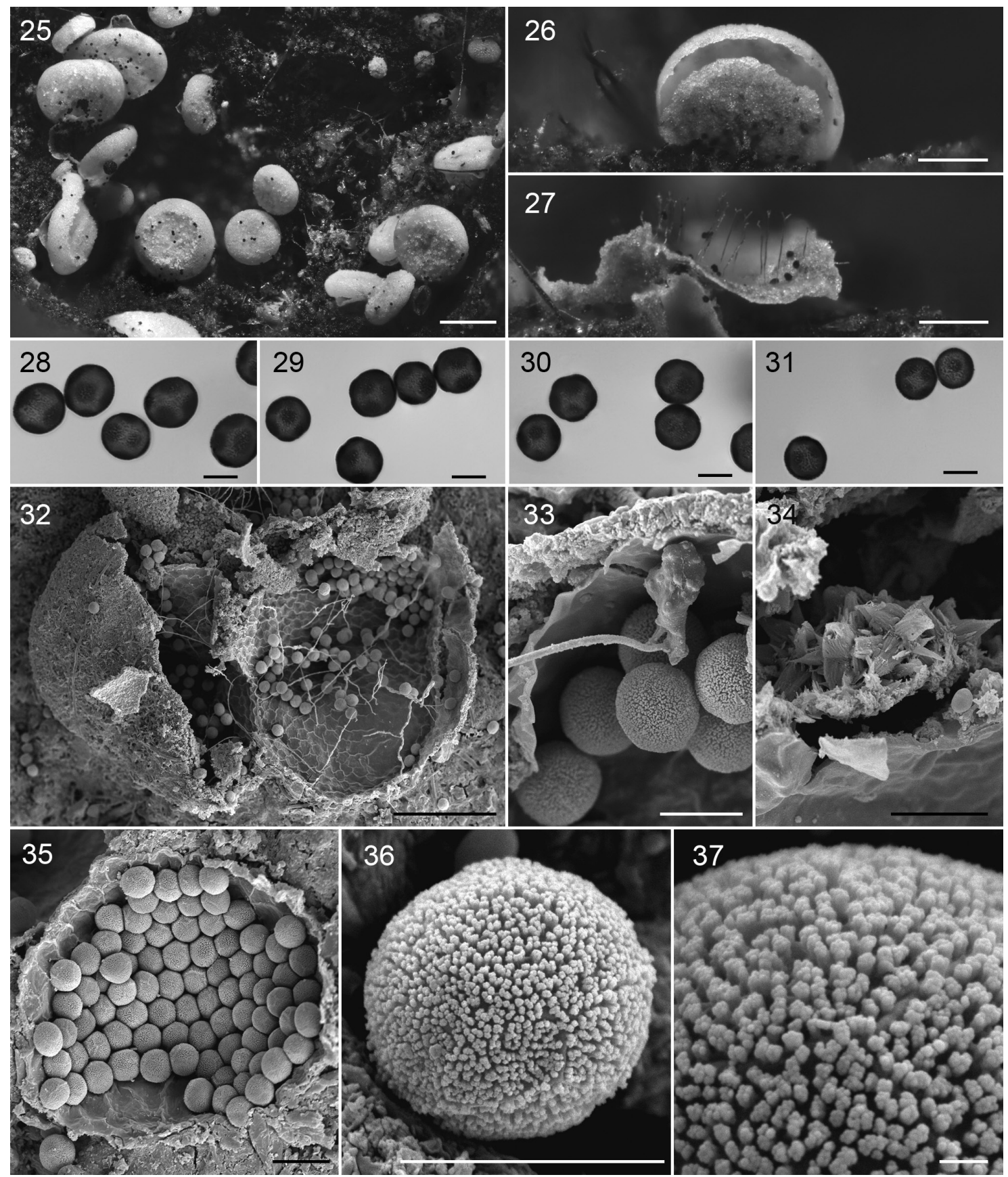

Figs. 25-37. Didymium peruvianum. 25. Sporocarps showing the eggshell-like outer peridium (sls 28943 , holotype). 26. Detail of a dehisced sporocarp (sls 28943, holotype). 27. Open sporocarp showing capillitial threads (sls 28943, holotype). 28. Spores by LM with broad pale bands (sls 28941). 29-30. Spores by LM (sls 28943, holotype). 31. Spores by LM (Lado 21925). 32-37. SEM image (sls 28943, holotype). 32. Detail of an open sporocarp. 33. Detail of the inner peridial layer. 34. Crystals of the peridium. 35. Open sporocarp showing the arrangement of the spores. 36. Spore. 37. Detail of the dense ornamentation of the spore. Bar: $25=0.2 \mathrm{~mm} ; 26-27=0.1 \mathrm{~mm} ; 28-31,33-34,36=10 \mu \mathrm{m} ; 32=100 \mu \mathrm{m} ; 35=20 \mu \mathrm{m} ; 37=1 \mu \mathrm{m}$.

Deuterochonia longipetala leaf bases (mc, pH 7.84), dwb 3676 (MA-Fungi 88436). PER-13-32: Furcraea occidentalis leaves, Lado 22658 (MA-Fungi 88034), Lado 22662 (MA-Fungi 88039).

A species widely distributed throughout the Neotropics and usually associated with succulent plants. In Peru previously reported from Cuzco (Rojas \& al., 2011).

\section{*Didymium verrucosporum A.L. Welden}

PER-13-32: herb litter, Lado 22645 (MA-Fungi 88021).
Widely distributed throughout the Neotropics, but not reported previously from Peru.

*Didymium wildpretii Mosquera, Estrada, Beltrán-

Tej., D. Wrigley \& Lado

PER-12-17: Tillandsia purpurea leaf bases (mc, pH 6.01), dwb 3535 (MA-Fungi 88421). PER-12-47: Tillandsia purpurea leaf bases (mc, $\mathrm{pH}$ 7.43), dwb 3564. PER-12-49: Ephedra americana twigs (mc, $\mathrm{pH} 5.99$ ), dwb 3627; Ephedra americana twigs (mc, pH 6.34), dwb 3629; Ephedra americana twigs (mc, pH 5.72), dwb 3630. PER-12-52: Tillandsia 
purpurea litter (mc, pH 6.13), dwb 3538; Tillandsia purpurea litter (mc, $\mathrm{pH}$ 5.20), dwb 3563; Tillandsia purpurea litter (mc, $\mathrm{pH}$ 6.41), dwb 3570 .

This species is usually associated with xeric environments often developing on the remains of cacti and other desert plants. These collections are typical. The sporothecae are covered with pale yellow to white lime crystals, sporocarps have short calcareous stalks, and spores 8-10 $\mu \mathrm{m}$ densely and uniformly warted. In the Neotropics previously reported from Mexico (Lado \& al., 2007; Estrada-Torres \& al., 2009; Esqueda \& al., 2011; Wrigley de Basanta \& al., 2015), Argentina (Lado \& al., 2011) and Chile (Lado \& al., 2013). These are the first records for Peru.

\section{*Echinostelium apitectum K.D. Whitney}

PER-14-26: Capparis scabrida bark (mc, pH 6.00), dwb 3694; Capparis scabrida bark (mc, pH 5.84), dwb 3698; Capparis scabrida bark (mc, pH 6.27), dwb 3709 .

In South America it was only known from Ecuador (McHugh, 2005) and Argentina (Lado \& al., 2014).

*Echinostelium arboreum H.W. Keller \& T.E. Brooks

PER-13-10: Melocactus peruviana litter (mc, pH 8.13), dwb 3648; Deuterochonia longipetala leaf bases (mc, pH 7.78), dwb 3650 (MA-Fungi 88425); Deuterochonia longipetala leaf bases (mc, $\mathrm{pH} 7.93$ ), dwb 3652; Deuterochonia longipetala leaf bases (mc, $\mathrm{pH}$ 7.66), dwb 3674. PER-14-06: Prosopis sp. bark (mc, pH 6.40), dwb 3711.

These are very large collections of this minute but very distinctive species. The sporocarps have a yellowish persistent shiny peridium, wide collar and spores $7-8 \mu \mathrm{m}$ in diameter. In South America, it is only known from Brazil (Cavalcanti, 2002), Ecuador (McHugh, 2005), Paraguay (McHugh, 2009) and Argentina (Lado \& al., 2011).

\section{*Echinostelium colliculosum K.D. Whitney \& H.W. Keller}

PER-13-10: Deuterochonia longipetala leaf bases (mc, $\mathrm{pH} 7.66$ ), dwb 3645. PER-13-12: Deuterochonia longipetala leaf bases (mc, $\mathrm{pH} 7.78$ ), dwb 3647. PER-14-06: Prosopis sp. bark (mc, pH 5.38), dwb 3700; Prosopis sp. bark (mc, pH 6.40), dwb 3701; Prosopis sp. bark (mc, pH 4.81), dwb 3712.

In South America previously reported from Brazil (Cavalcanti, 2002), Paraguay (McHugh, 2009), Chile (Lado \& al., 2013) and Argentina (Wrigley de Basanta \& al., 2010b; Lado \& al., 2014).

\section{Echinostelium minutum de Bary}

PER-13-10: Puya sp. inflorescence (mc, pH 5.3), aet 13097.

Widely distributed throughout the Neotropics. In Peru previously reported from Loreto (Wrigley de Basanta \& al., 2008a).

\section{*Enerthenema papillatum (Pers.) Rostaf.}

PER-12-34: mixed Tillandsia (mc, pH 5.68), sls 28323; mixed Tillandsia (mc, pH 5.71), sls 28325.

Widely distributed throughout the Neotropics, but not reported previously from Peru.

\section{*Fuligo cinerea (Schwein.) Morgan}

PER-14-42: Capparis scabrida leaves, Lado 23587 (MA-Fungi 88376), Lado 23590 (MA-Fungi 88379), Lado 23591 (MA-Fungi 88380).

Widely distributed throughout the Neotropics. These collections were found in the North of the country, in dunes fixed by Capparis scabrida. The spores by SEM (Fig. 39) show the chracteristic ornamentation of warts connected by minute crests in a reticulate pattern.

\section{Fuligo septica (L.) F.H. Wigg. Fig. 39.}

PER-14-10: Capparis scabrida leaves, Lado 23414 (MA-Fungi 88078). PER14-41: Capparis scabrida leaves, Lado 23579 (MA-Fungi 88367).

In Peru previously reported from Madre de Dios (Rojas $\&$ al., 2011).

\section{*Hemitrichia serpula (Scop.) Rostaf. ex Lister}

PER-14-34: wood, Lado 23560 (MA-Fungi 88348).

Widely distributed throughout the Neotropics, but not reported previously from Peru.

\section{${ }^{\circ}$ Licea bulbosa Nann.-Bremek. \& Y. Yamam.}

PER-14-26: Capparis scabrida bark (mc, pH 6.00), dwb 3693; Capparis scabrida bark (mc, $\mathrm{pH}$ 6.27), dwb 3710.

In the Neotropics this species was only known previously from Cuba (Wrigley de Basanta \& Lado, 2005; Camino $\&$ al., 2008). It is easily recognised by the transparent base to the sporotheca with prominent ornamentation on the edge, and the smooth spores 10-11 $\mu \mathrm{m}$ diam.

\section{* Licea eremophila D. Wrigley, Lado \& Estrada}

PER-12-47: Tillandsia purpurea leaf bases (mc, pH 7.43), dwb 3541 (MA-Fungi 88422).

The peridium of this tiny yellow species breaks into platelets. It has polyhedral yellow spores 11-12 $\mu \mathrm{m}$ diam. with a uniform thick wall and each sporocarp forms from a colourless to yellow protoplasmodium. In South America only known from the arid lands of Argentina (Wrigley de Basanta \& al., 2010; Lado \& al., 2011) and Chile (Wrigley de Basanta \& al., 2010a; Lado \& al., 2013).

\section{*Licea succulenticola Mosquera, Lado, Estrada \& Beltrán-Tej.}

PER-13-32: Furcraea occidentalis leaves, Lado 22658 (MA-Fungi 88035).

This species usually develops on succulent plants, but has also been found on leaves and leaf bases. It is distributed throughout the arid regions of Mexico (Mosquera \& al., 2003; Lado \& al., 2007; Moreno \& al., 2007; Estrada-Torres \& al., 2009; Salazar-Márquez \& al., 2014; Lizárraga \& al., 2015), Brazil (Bezerra \& al., 2014), Ecuador (McHugh, 2005), Chile (Lado \& al., 2007, 2013), Argentina (Lado \& al., 2011; 2014) and Paraguay (McHugh, 2009). 

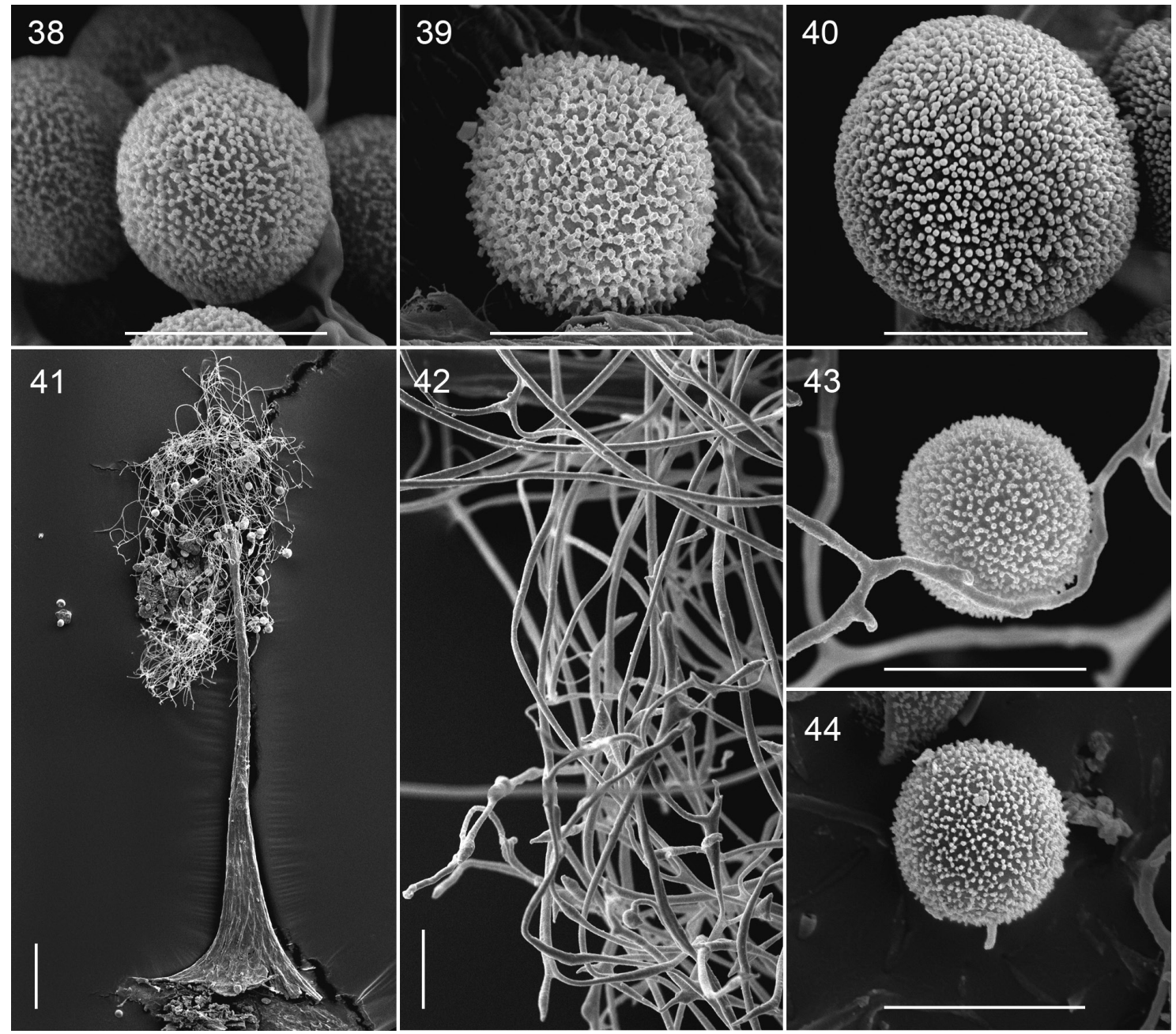

Figs. 38-44. SEM images. 38. Didymium dubium (Lado 22554), spore. 39. Fuligo cinerea (Lado 23587), spore. 40-42. Paradiacheopsis fimbriata (Lado 21965). 40. Spore. 41. Sporocarp. 42. Detail of the capillitial threads. 43-44. Stemonitopsis gracilis (Lado 23429), spores. Bar: 38-40, 42-44=10 $\mu$ m; $41=100 \mu \mathrm{m}$.

*Metatrichia vesparia (Batsch) Nann.-Bremek. ex G.W. Martin \& alexop.

PER-14-34: wood, Lado 23562 (MA-Fungi 88350), Lado 23563 (MA-Fungi 88351).

Widely distributed throughout the Neotropics but not previously reported from Peru.

*Paradiacheopsis fimbriata (G. Lister \& Cran) Hertel ex Nann.-Bremek. Figs. 40-42.

PER-12-33: mixed Tillandsia (mc, pH 6.47), sls 28938. PER-12-34: Tillandsia latifolia leaves, Lado 21965 (MA-Fungi 87781). PER-12-49: Ephedra americana twigs (mc, pH 5.1), aet 12233.

In South America only previously recorded from Ecuador (Schnittler \& al., 2002) and Chile (Lado \& al., 2013). The collection Lado 21965, illustrated in Figs. 40-42 is a little aberrant. The capillitium arises along the length of the columella and is unusually abundant, the threads are smooth, the diameter uniform and the ends are club-shaped, but not as obviously as in the typical specimens. The spores are larger, 12-15 $\mu \mathrm{m}$ diam (11-13 $\mu \mathrm{m}$ diam in Nannenga-Beremekamp,
1991), but densely covered with fine warts (Fig. 40) as described in the literature.

\section{Perichaena chrysosperma (Curr.) Lister}

PER-12-26: aerial litter (mc, pH 5.60), sls 29999. PER-14-18: wood, Lado 23511 (MA-Fungi 88306).

Widely distributed throughout the Neotropics. In Peru previously reported from Madre de Dios (Rojas \& al., 2011).

\section{Perichaena depressa Lib.}

PER-12-28: branch of an Asteraceae, Lado 21924 (MA-Fungi 87744); twigs (mc, pH 6.60), sls 28937. PER-12-29: ground litter (mc, $\mathrm{pH} 7.70$ ), sls 30103. PER-12-48: Haageocereus sp. remains (mc, pH 7.4), aet 12359 PER-12-49: Ephedra americana twigs (mc, $\mathrm{pH} 5.1$ ), aet 12357. PER-13-32: Furcraea occidentalis leaves, Lado 22661 (MA-Fungi 88038). PER-14-18 bark, Lado 23509 (MA-Fungi 88303). PER-14-19: Agave americana leaves, Lado 23516 (MA-Fungi 88311), Lado 23517 (MA-Fungi 88312).

In Peru previously reported from Cuzco, Lima and Madre de Dios (Rojas \& al., 2011). A common species throughout the Neotropics. 


\section{*Perichaena dictyonema Rammeloo}

PER-12-17: ground litter (mc, pH 4.79), sls 30195.

In South America this species was only known from Ecuador (Schnittler \& Stephenson, 2002; Schnittler \& al., 2002; Stephenson \& al., 2004; Mchugh, 2005; Wrigley de Basanta \& al., 2008a; Poulain \& al., 2011) and Paraguay (McHugh, 2009). The collection consists of about 20 sporocarps and is absolutely typical in all respects.

\section{*Perichaena luteola (Kowalski) Gilert}

PER-12-48: Haageocereus sp. remains (mc, pH 7.4), aet 12360.

This is the second record for South America. This species was previously reported only from Argentina (Lado \& al., 2014).

\section{*Perichaena quadrata T. Macbr.}

PER-13-10: Deuterochonia longipetala leaf bases (mc, pH 7.66), dwb 3671. PER-13-28: twigs, Lado 22616 (MA-Fungi 87995). PER-13-32: Furcraea occidentalis leaves, Lado 22660 (MA-Fungi 88037). PER-14-06: Prosopis sp. bark (mc, pH 6.40), dwb 3730. PER-14-19: Agave americana leaves, Lado 23514 (MA-Fungi 88308), Lado 23515 (MA-Fungi 88309). PER-14-20: liana, Lado 23523 (MA-Fungi 88318).

In South America only known from Argentina (Lado \& al., 2011, 2014) and Chile (Lado \& al., 2013).

\section{${ }^{\circ}$ Perichaena tessellata G. Lister aff.}

PER-13-19: Capparis scabrida leaf litter (mc, pH 7.71), dwb 3678. PER-1411: Capparis scabrida leaf litter (mc, pH 7.84), dwb 3697.

These collections on the same substrate, but from two separate years and different places, show a partially chequered peridium. The small, scattered, sessile sporocarps are less than $0.5 \mathrm{~mm}$ diam. The yellowish peridium breaks up into irregular sized platelets. The capillitium is scanty, the threads of 2-3 $\mu \mathrm{m}$ diam., irregular, with constrictions and ornamented with warts. The spores however are larger than those described by Lister (1931), from 12-14(-15) $\mu \mathrm{m}$ diam. vs 9-10 $\mu \mathrm{m}$ diam., yellow, and thick walled but with a thinner area to the wall. The sporocarp colour is also lighter than the description of $P$. tesselata, yellowish brown not purplish black. The Peruvian material is apparently the closest to this species, but only further collections can confirm if it is the same. In the Neotropics it was only reported from El Salvador (Rojas \& al., 2013), and is otherwise a rare species. If confirmed these collections would be the first records of the species in South America.

\section{Perichaena vermicularis (Schwein.) Rostaf.}

PER-12-26: aerial litter (mc, pH 5.66), sls 30200; aerial litter (mc, pH 5.27), sls 30219. PER-12-28: branch of an Asteraceae, Lado 21920 (MA-Fungi 87743). PER-12-29: twigs and wood of an Asteraceae, Lado 21942 (MA-Fungi 87761). PER-12-32: Tillandsia sp. inflorescence (mc, $\mathrm{pH}$ 6.9), aet 12236, (mc, $\mathrm{pH}$ 6.9), aet 12289. PER-12 34: Washingtonia sp. leaves, Lado 21992 (MA-Fungi 87804); mixed Tillandsia (mc, $\mathrm{pH}$ 5.71), sls 28313; mixed Tillandsia (mc, pH 4.83), sls 28321. PER-12-41: Corryocactus brachypetalus remains, Lado 22024 (MA-Fungi 87817). PER-12-42: Trixis cacalioides twigs, Lado 22050 (MA-Fungi 87839); Alternantera pubiflora twigs and leaves, Lado 22034 (MA-Fungi 87827). PER-12-43: twigs, Lado 22058 (MA-Fungi 87843), Lado 22062 (MA-Fungi 87848), Lado 22076 (MA-Fungi 87862), Lado
22078 (MA-Fungi 87864), Lado 22081 (MA-Fungi 87868), Lado 22083 (MA-Fungi 87872), Lado 22084 (MA-Fungi 87873), Lado 22090 (MA-Fungi 87881); Heliotropium arborescens twigs, Lado 22093 (MA-Fungi 87886). PER-13-18: Capparis scabrida leaf litter (mc, pH 8.02), dwb 3649 (MA-Fungi 88424); Capparis scabrida leaf litter (mc, $\mathrm{pH}$ 7.77), dwb 3654 (MA-Fungi 88427); Capparis scabrida leaf litter (mc, $\mathrm{pH}$ 7.66), dwb 3656 (MA-Fungi 88429); Capparis scabrida twigs and leaves, Lado 22494 (MA-Fungi 87920), Lado 22504 (MA-Fungi 87931). PER13-19: Capparis scabrida leaf litter (mc, $\mathrm{pH}$ 8.05), dwb 3663; Capparis scabrida leaf litter (mc, pH 7.71), dwb 3668 (MA-Fungi 88435). PER-13. 28: twigs, Lado 22614 (MA-Fungi 87993). PER-13-32: Arundo sp. litter, Lado 22644 (MA-Fungi 88020). PER-14-11: Capparis scabrida leaf litter (mc, pH 7.84), dwb 3706. PER-14-42: Capparis scabrida leaves, Lado 23589 (MA-Fungi 88378). PER-14-43: Capparis scabrida leaves, Lado 23618 (MA-Fungi 88414).

Widely distributed throughout the Neotropics. In Peru previously reported from Loreto (Wrigley de Basanta \& al., 2008a). The moist chamber collections of this species on Capparis scabrida were very extensive, sometimes covering almost all the substrate in a $9 \mathrm{~cm}$ culture dish.

*Physarum aeneum (Lister) R.E. Fr. Figs. 51-52.

PER-13-28: twigs, Lado 22615 (MA-Fungi 87994).

Distributed throughout the Neotropics. The spores by SEM of the unique collection from Peru are illustrated in Figs. 51-52. By LM are typical, pale brown, 8-9 $\mu \mathrm{m}$ diam, and minutely warted, the warts by SEM are scanty and well defined (Figs. 51-52).

\section{Physarum album (Bull.) Chevall.}

PER-13-27: twigs and litter under Schinus molle, Lado 22611 (MA-Fungi 87989).

Widely distributed throughout the Neotropics. In Peru previously reported from Madre de Dios (Rojas \& al., 2011).

\section{*Physarum atacamense D. Wrigley, Lado \& Estrada}

PER-12-17: Tillandsia purpurea leaf bases (mc, pH 5.61), dwb 3543 PER-12-26: Tillandsia sp. inflorescence (mc, pH 5.5), aet 12254 (mc, $\mathrm{pH}$ 5.5), aet 12291; ground litter (mc, pH 4.50), sls 28428 (MA-Fungi 88445). PER-12-33: Tillandsia sp. litter (mc, pH 5.78), dwb 3560. PER-12-34: Phoenix sp. rachis, Lado 21989 (MA-Fungi 87802), Lado 21990 (MA-Fungi 87803). PER-12-35: Haageocereus acranthus flowers (mc, pH 7.0), aet 12238, (mc, $\mathrm{pH} 7.0$ ), aet 12239b, (mc, $\mathrm{pH} 8.0$ ), aet 12244. PER-12-52: Tillandsia purpurea litter (mc, pH 5.20), dwb 3562. PER-13-10: Puya sp. inflorescence (mc, pH 5.3), aet 13083; Puya sp. leaves, Lado 22393 (MA-Fungi 87905), Lado 22395 (MA-Fungi 87907). PER-13-12: Deuterochonia longipetala leaf bases (mc, $\mathrm{pH}$ 7.68), dwb 3669; Deuterochonia longipetala leaf bases (mc, pH 7.78), dwb 3670; Deuterochonia longipetala inflorescence (mc, pH 5.6), aet 13067. PER13-15: Tillandsia sp. litter, Lado 22420 (MA-Fungi 87918), Lado 22421 (MA-Fungi 87919). PER-13-18: Capparis scabrida leaf litter (mc, pH 8.02), dwb 3653 (MA-Fungi 88426); Capparis scabrida leaf litter (mc, $\mathrm{pH}$ 7.77), dwb 3655 (MA-Fungi 88428); Capparis scabrida leaf litter (mc, $\mathrm{pH}$ 7.66), dwb 3657 (MA-Fungi 88430); Capparis scabrida twigs and leaves, Lado 22494 (MA-Fungi 87921), Lado 22495 (MA-Fungi 87922), Lado 22496 (MA-Fungi 87923), Lado 22498 (MA-Fungi 87925), Lado 22499 (MA-Fungi 87926), Lado 22500 (MA-Fungi 87927), Lado 22502 (MA-Fungi 87929), Lado 22503 (MA-Fungi 87930), Lado 22505 (MA-Fungi 87933), Lado 22506 (MA-Fungi 87934), Lado 22507 (MA-Fungi 87936); Capparis scabrida fruit, Lado 22508 (MA-Fungi 87937); Capparis scabrida twigs and leaves, Lado 22509 (MA-Fungi 87938), Lado 22510 (MA-Fungi 87939), Lado 22511 (MA-Fungi 87940), Lado 22512 (MA-Fungi 87941). PER-13-19: Capparis scabrida leaf litter (mc, pH 7.74), dwb 3658 (MA-Fungi 88431); Capparis scabrida 
leaf litter (mc, pH 8.05), dwb 3662 (MA-Fungi 88433); Capparis scabrida leaf litter (mc, pH 7.71), dwb 3667 (MA-Fungi 88434); Capparis scabrida leaves, Lado 22513 (MA-Fungi 87942), Lado 22514 (MA-Fungi 87943), Lado 22516 (MA-Fungi 87944), Lado 22517 (MA-Fungi 87945), Lado 22518 (MA-Fungi 87946), Lado 22520 (MA-Fungi 87948), Lado 22521 (MA-Fungi 87949). PER-13-20: leaf litter, Lado 22525 (MA-Fungi 87953), Lado 22528 (MA-Fungi 87956), Lado 22529 (MA-Fungi 87957), Lado 22531 (MA-Fungi 87959). PER-13-29: Capparis scabrida twigs and leaves, Lado 22618 (MA-Fungi 87997), Lado 22619 (MA-Fungi 87998), Lado 22620 (MA-Fungi 87999). PER-13-30: Tillandsia sp. inflorescence (mc, $\mathrm{pH} 4.8$ ), aet 13084, (mc, $\mathrm{pH} 4.5$ ), aet 13100. PER-13-31: Armatocereus procerus litter (mc, $\mathrm{pH}$ 8.03), dwb 3690; Capparis ovalifolia leaves, Lado 22622 (MA-Fungi 88000), Lado 22624 (MA-Fungi 88002), Lado 22630 (MA-Fungi 88007), Lado 22634 (MA-Fungi 88011). PER-14-02: Capparis scabrida leaf litter (mc, $\mathrm{pH} 7.80$ ), dwb 3703; Capparis scabrida leaves, Lado 23382 (MA-Fungi 88050), Lado 23383 (MA-Fungi 88051); Capparis scabrida fruit, Lado 23384 (MA-Fungi 88052); Capparis scabrida leaves, Lado 23385 (MA-Fungi 88053), Lado 23386 (MA-Fungi 88054), Lado 23387 (MA-Fungi 88055), Lado 23388 (MA-Fungi 88056). PER-14-03: Capparis scabrida leaf litter (mc, pH 8.03), dwb 3705; Capparis scabrida leaves, Lado 23389 (MA-Fungi 88057), Lado 23391 (MA-Fungi 88058), Lado 23395 (MA-Fungi 88059), Lado 23398 (MA-Fungi 88062), Lado 23400 (MA-Fungi 88064). PER-14-04: Capparis scabrida leaves, Lado 23401 (MA-Fungi 88065). PER-14-05: Capparis scabrida leaves, Lado 23402 (MA-Fungi 88066), Lado 23403 (MA-Fungi 88068); Capparis avicenifolia twigs and leaves, Lado 23404 (MA-Fungi 88069); Capparis scabrida leaves, Lado 23405 (MA-Fungi 88070), Lado 23406 (MA-Fungi 88071), Lado 23407 (MA-Fungi 88072). PER-14-06: Prosopis sp. bark (mc, pH 5.38), dwb 3726. PER-14-07: Capparis avicenifolia leaf litter (mc, $\mathrm{pH}$ 8.03), dwb 3721; Capparis avicenifolia leaves, Lado 23408 (MA-Fungi 88073), Lado 23409 (MA-Fungi 88074). PER-14-08: Capparis avicenifolia leaf litter (mc, $\mathrm{pH} 7.45$ ), dwb 3722; Capparis avicenifolia twigs and leaves, Lado 23410 (MA-Fungi 88075), Lado 23411 (MA-Fungi 88076). PER-14-09: Capparis avicenifolia leaf litter (mc, $\mathrm{pH}$ 7.49), dwb 3714; Capparis avicenifolia leaves, Lado 23412 (MA-Fungi 88077). PER-14-10 Capparis scabrida leaves, Lado 23415 (MA-Fungi 88079); Capparis avicenifolia twigs and leaves, Lado 23416 (MA-Fungi 88080). PER-14-11: Capparis scabrida leaf litter (mc, $\mathrm{pH}$ 7.84), dwb 3707; Capparis scabrida leaves, Lado 23419 (MA-Fungi 88083), Lado 23421 (MA-Fungi 88085), Lado 23422 (MA-Fungi 88086), Lado 23423 (MA-Fungi 88087), Lado 23424 (MA-Fungi 88088). PER-14-29: Armatocereus sp. remains, Lado 23540 (MA-Fungi 88330), Lado 23542 (MA-Fungi 88331), Lado 23543 (MA-Fungi 88332), Lado 23544 (MA-Fungi 88333), Lado 23545 (MA-Fungi 88334). PER-14-38: Neoraimondia sp. remains, Lado 23569 (MA-Fungi 88357). PER-14-39: Armatocereus sp. remains, Lado 23570 (MA-Fungi 88358), Lado 23572 (MA-Fungi 88360), Lado 23573 (MA-Fungi 88361), Lado 23574 (MA-Fungi 88362). PER-14-41: Capparis scabrida leaves, Lado 23580 (MA-Fungi 88368); Capparis ovalifolia leaves, Lado 23581 (MA-Fungi 88370); Capparis ovalifolia twigs and leaves, Lado 23582 (MA-Fungi 88371); Capparis ovalifolia leaves, Lado 23583 (MA-Fungi 88372), Lado 23584 (MA-Fungi 88373), Lado 23585 (MA-Fungi 88374), Lado 23586 (MA-Fungi 88375). PER-14-42: twigs and leaves of Capparis ovalifolia, Lado 23592 (MA-Fungi 88381), Lado 23593 (MA-Fungi 88382); Capparis scabrida leaves, Lado 23594 (MA-Fungi 88383); Capparis ovalifolia leaves, Lado 23595 (MA-Fungi 88385), Lado 23596 (MA-Fungi 88387); twigs and leaves of Capparis ovalifolia, Lado 23600 (MA-Fungi 88392), Lado 23601 (MA-Fungi 88393), Lado 23603 (MA-Fungi 88397). PER-14-43: Capparis ovalifolia leaves, Lado 23607 (MA-Fungi 88401), Lado 23608 (MA-Fungi 88402), Lado 23609 (MA-Fungi 88403), Lado 23610 (MA-Fungi 88404), Lado 23611 (MA-Fungi 88405), Lado 23612 (MA-Fungi 88407); Capparis scabrida leaves, Lado 23613 (MA-Fungi 88408), Lado 23616 (MA-Fungi 88411), Lado 23617 (MA-Fungi 88412), Lado 23618 (MA-Fungi 88413), Lado 23619 (MA-Fungi 88415).

Only previously known from the Atacama Desert in Chile (Wrigley de Basanta \& al., 2012). These records expand the distribution of the species to the coastal arid lands of Peru, where it was almost the most frequently recorded species, 135 collections. The collections were extensive both in the field and in moist chamber cultures. From these numerous collections, the description of the species can be broadened to include some sessile or very short stalked sporocarps, the stalk in some cases is flat, entirely yellow, not darker at the base, longitudinally striate and common to several sporocarps.

\section{*Physarum bitectum G. Lister}

PER-12-19: aerial twigs (mc, pH 5.52), sls 29170.

The single collection was typical in all respects.

\section{Physarum bogoriense Racib.}

PER-13-32: leaves, Lado 22642 (MA-Fungi 88019). PER-14-18: leaf litter, Lado 23506 (MA-Fungi 88300).

Widely distributed throughout the Neotropics. In Peru previously reported from Huánuco and Loreto (Whitney \& Keller, 1982; Wrigley de Basanta \& al., 2008a).

\section{*Physarum cinereum (Batsch) Pers.}

PER-12-29: twigs of an Asteraceae, Lado 21943 (MA-Fungi 87762); wood of an Asteraceae, Lado 21947 (MA-Fungi 87767). PER-12-34: Tillandsia purpurea inflorescence (mc, pH 7.0), aet 12246.

Widely distributed throughout the Neotropics, but not previously reported from Peru.

\section{*Physarum cf. clavisporum G. Moreno, A. Sánchez, A. Castillo \& Illana Figs. 45-50.}

PER-13-12: Haageocereus sp. remains, Lado 22417 (MA-Fungi 87915) PER-13-19: Capparis scabrida leaves, Lado 22519 (MA-Fungi 87947). PER-13-20: leaf litter, Lado 22531 (MA-Fungi 87960). PER-13-28: Espostoa sp. remains, Lado 22613 (MA-Fungi 87992). PER-13-31: Capparis ovalifolia leaves, Lado 22623 (MA-Fungi 88001), Lado 22626 (MA-Fungi 88003), Lado 22627 (MA-Fungi 88004), Lado 22628 (MA-Fungi 88005), Lado 22629 (MA-Fungi 88006), Lado 22631 (MA-Fungi 88008), Lado 22632 (MA-Fungi 88009), Lado 22633 (MA-Fungi 88010). PER-14-02: Capparis scabrida leaf litter (mc, $\mathrm{pH}$ 7.80), dwb 3704. PER-14-03: Capparis scabrida wood, Lado 23396 (MA-Fungi 88060); Capparis scabrida leaves, Lado 23397 (MA-Fungi 88061), Lado 23399 (MA-Fungi 88063). PER-14-05: Capparis scabrida leaves, Lado 23403 (MA-Fungi 88067). PER-14-11: Capparis scabrida leaves, Lado 23417 (MA-Fungi 88081), Lado 23418 (MA-Fungi 88082), Lado 23420 (MA-Fungi 88084). PER-14-29: Armatocereus sp. remains, Lado 23539 (MA-Fungi 88329). PER-14-41: Capparis scabrida leaves, Lado 23580 (MA-Fungi 88369). PER-14-42: Capparis ovalifolia leaves, Lado 23595 (MA-Fungi 88386), Lado 23596 (MA-Fungi 88388), Lado 23597 (MA-Fungi 88389); twigs and leaves of Capparis ovalifolia, Lado 23598 (MA-Fungi 88390), Lado 23599 (MA-Fungi 88391), Lado 23601 (MA-Fungi 88394), Lado 23602 (MA-Fungi 88395), Lado 23603 (MA-Fungi 88396), Lado 23604 (MA-Fungi 88398); Capparis scabrida leaves, Lado 23605 (MA-Fungi 88399). PER-14-43: Capparis ovalifolia leaves, Lado 23606 (MA-Fungi 88400), Lado 23611 (MA-Fungi 88406); Capparis scabrida leaves, Lado 23614 (MA-Fungi 88409).

Second record for South America, previously reported only from Chile (Lado \& al., 2013). Again this is a rare species with a restricted distribution that is appears abundantly in the Peruvian coastal desert. Macroscopically, the Peruvian specimens are almost indistinguishable from $P$. bitectum, but detailed observation of the spores reveals disperse prominent spines in P. clavisporum (Figs. 46-47) and uniformly distributed warts in P. bitectum. Our collections agree, in general, with the original description of the species (Moreno \& al., 2009), since the spores have the typical dark purple brown colour with a paler area, bearing dispersed and prominent 

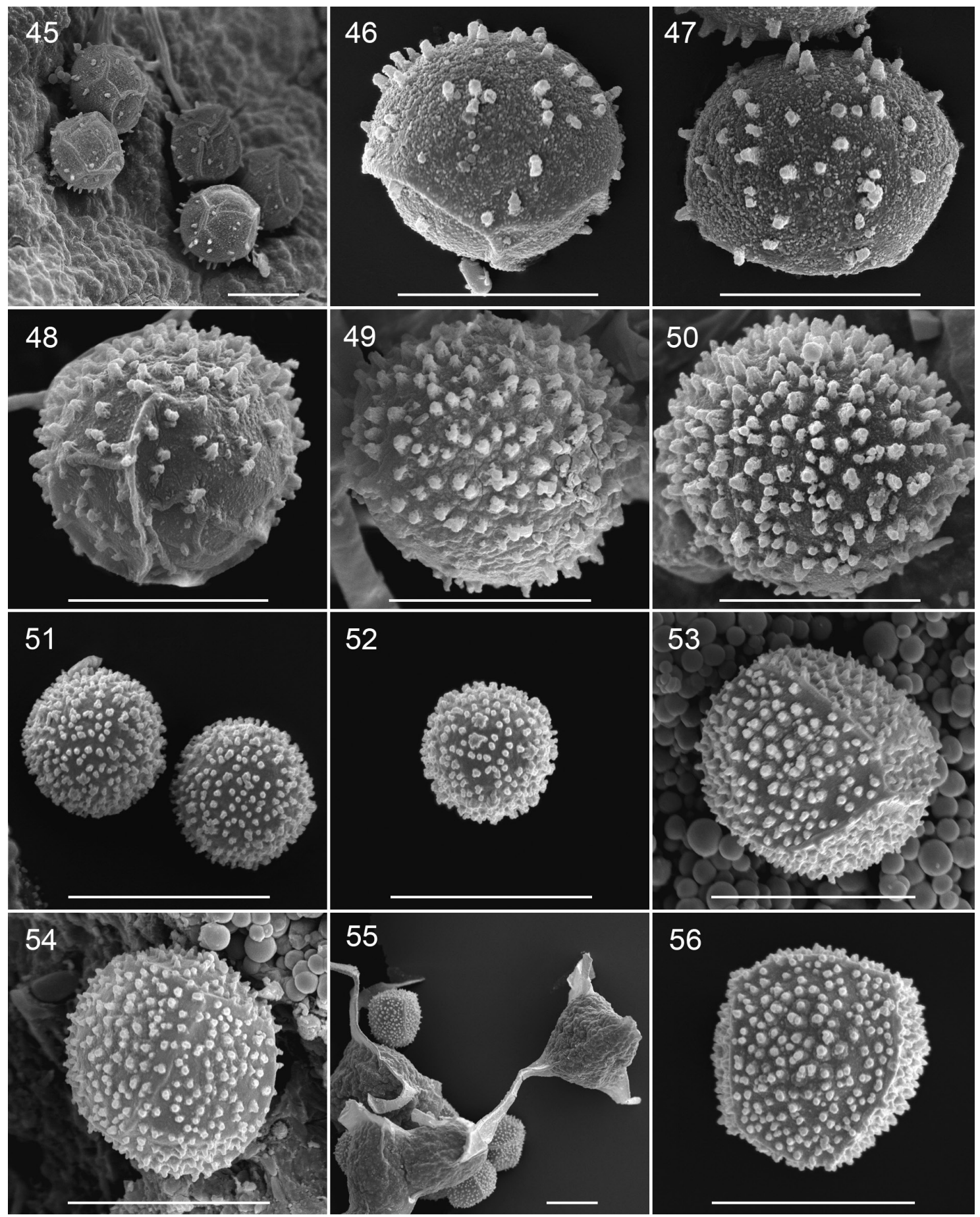

Figs. 45-56. SEM images. 45-47. Physarum cf. clavisporum (Lado 22417), spores. 48-50. Physarum cf. clavisporum (Lado 23399), spores. 51-52. Physarum aeneum (Lado 22615), spores. 53-56. Physarum spectabile (Lado 22659), spores and detail of the capillitium. Bar: 45-56=10 $\mu$ m.

spines, and the spore surface covered by tiny densely distributed warts visible only by SEM (Figs. 45-50). But the spines of the spores by SEM, in our collections, are formed of spines or bacula (Figs. 45-50) instead of the pila with widened to slightly coralloid apex of the type material. In addition, in the Peruvian material a gradation in the quantity and density of the spines, from few and dispersed (Figs. 45-48) to abundant and regularly distributed (Figs. 49-50) can be observed, differentiating this material from that described by Moreno \& al. (2009), and for that reason the collections have been left as cf. They may indicate an ecotype or variety of the species, but further investigation is needed to confirm or refute this.

\section{Physarum compressum Alb. \& Schwein.}

PER-12-34: Tillandsia latifolia inflorescence (mc, pH 7.2), aet 12234 , (mc, $\mathrm{pH} 7.2$ ), aet 12242, (mc, $\mathrm{pH}$ 6.2), aet 12310; Tillandsia purpured inflorescence (mc, pH 7.0), aet 12231a, (mc, pH 7.0), aet 12284, 
(mc, pH 7.0), aet 12287, (mc, pH 7.3), aet 12306; mixed Tillandsia (mc, $\mathrm{pH}$ 4.83), sls 28357; mixed Tillandsia (mc, $\mathrm{pH}$ 5.68), sls 28949. PER12-35: Haageocereus acranthus flowers (mc, $\mathrm{pH} 8.1$ ), aet 12245, (mc, $\mathrm{pH} 8.1$ ), aet 12249 (mc, $\mathrm{pH} 8.1$ ), aet 12261, (mc, pH 7.0), aet 12263. PER-13-27: aerial litter (mc, pH 6.62), sls 31277; ground litter (mc, $\mathrm{pH}$ 7.50), sls 31148. PER-13-32: Furcraea occidentalis leaves, Lado 22655 (MA-Fungi 88031), Lado 22657 (MA-Fungi 88033), Lado 22659 (MA-Fungi 88036), Lado 22663 (MA-Fungi 88040). PER-14-18: bark, Lado 23510 (MA-Fungi 88304). PER-14-36: Armatocereus sp. remains, Lado 23568 (MA-Fungi 88356).

Widely distributed throughout the Neotropics. In Peru previously reported from Loreto (Wrigley de Basanta \& al., 2008a). Collection sls 31148 has been left as cf. since the collection was poorly developed, but it appeared to fit this species in most respects.

\section{*Physarum javanicum Racib.}

PER-12-29: twigs and leaves of an Asteraceae, Lado 21929 (MA-Fungi 87747), Lado 21930 (MA-Fungi 87748); Schinus molle wood, Lado 21941 (MA-Fungi 87760). PER-13-27: twigs and litter under Schinus molle, Lado 22611 (MA-Fungi 87990). PER-13-32: herb litter, Lado 22641 (MA-Fungi 88018).

A species widely distributed throughout the Neotropics, but not previously reported from Peru.

\section{*Physarum licheniforme (Schwein.) Lado}

PER-14-19: Furcraea andina leaves, Lado 23512 (MA-Fungi 88307).

In South America only previously known from Argentina (Lado \& al., 2011, 2014) and Chile (Lado \& al., 2013).

\section{Physarum melleum (Berk. \& Broome) Massee}

PER-13-27: twigs and litter under Schinus molle, Lado 22608 (MA-Fungi 87986), Lado 22609 (MA-Fungi 87987), Lado 22610 (MA-Fungi 87988).

Widely distributed throughout the Neotropics. In Peru previously reported from Loreto (Wrigley de Basanta \& al., 2008a).

\section{*Physarum notabile T. Macbr.}

PER-12-34: Washingtonia sp. leaves, Lado 21994 (MA-Fungi 87806), Lado 21995 (MA-Fungi 87808). PER-12-39: Acacia twigs (mc, pH 6.05), sls 28358 (MA-Fungi 88443). PER-12-42: Trixis cacalioides twigs, Lado 22048 (MA-Fungi 87837). PER-13-18: Capparis scabrida twigs and leaves, Lado 22497 (MA-Fungi 87924), Lado 22501 (MA-Fungi 87928), Lado 22504 (MA-Fungi 87932), Lado 22506 (MA-Fungi 87935). PER-13-20: twigs, Lado 22522 (MA-Fungi 87950). PER-14 11: Capparis scabrida leaf litter (mc, $\mathrm{pH}$ 7.84), dwb 3708. PER-14-42: Capparis scabrida leaves, Lado 23588 (MA-Fungi 88377), Lado 23594 (MA-Fungi 88384). PER-14-47: Neoraimondia sp. remains, Lado 23647 (MA-Fungi 88416).

Distributed throughout the Neotropics, but not previously reported from Peru.

\section{*Physarum cf. pezizoideum (Jungh.) Pavill. \& Lagarde \\ PER-14-06: Prosopis sp. bark (mc, pH 6.41), dwb 3696.} Brazil

\section{Physarum pusillum (Berk. \& M.A. Curtis) G. Lister}

PER-12-28: branch of an Asteraceae, Lado 21917 (MA-Fungi 87740), Lado 21926 (MA-Fungi 87745), Lado 21927 (MA-Fungi 87746). PER12-29: twigs and leaves of an Asteraceae, Lado 21934 (MA-Fungi 87751), Lado 21936 (MA-Fungi 87754), Lado 21937 (MA-Fungi 87756), Lado 21938 (MA-Fungi 87758), Lado 21946 (MA-Fungi 87766). PER-12

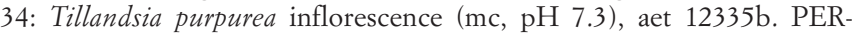
12-39: Acacia twigs (mc, pH 5.24), sls 28939. PER-12-41: Corryocactus brachypetalus remains, Lado 22023 (MA-Fungi 87816). PER-12-42: Alternantera pubiflora twigs and leaves, Lado 22030 (MA-Fungi 87823), Lado 22031 (MA-Fungi 87824); Trixis cacalioides twigs, Lado 22049 (MA-Fungi 87838). PER-12-43: twigs, Lado 22059 (MA-Fungi 87844), Lado 22061 (MA-Fungi 87846), Lado 22066 (MA-Fungi 87850), Lado 22071 (MA-Fungi 87856), Lado 22073 (MA-Fungi 87858), Lado 22080 (MA-Fungi 87866), Lado 22082 (MA-Fungi 87870), Lado 22084 (MA-Fungi 87874), Lado 22085 (MA-Fungi 87876); Heliotropium arborescens twigs, Lado 22093 (MA-Fungi 87887), Lado 22095 (MA-Fungi 87889); Lado 22067 (MA-Fungi 87851). PER-13-10: Deuterochonia longipetala leaf bases (mc, pH 7.66), dwb 3651; Deuterochonia longipetala leaf bases (mc, pH 7.93), dwb 3665; Puya sp. leaves, Lado 22394 (MA-Fungi 87906). PER-13-21: Neoraimondia sp. remains, Lado 22545 (MA-Fungi 87974), Lado 22546 (MA-Fungi 87975); twigs, Lado 22548 (MA-Fungi 87977), Lado 22550 (MA-Fungi 87979), Lado 22551 (MA-Fungi 87980). PER-13-36: Melocactus sp. remains, Lado 22755 (MA-Fungi 88046). PER-14-12: Neoraimondia sp. remains, Lado 23425 (MA-Fungi 88089), Lado 23427 (MA-Fungi 88091), Lado 23428 (MA-Fungi 88092), Lado 23430 (MA-Fungi 88094), Lado 23431 (MA-Fungi 88095), Lado 23433 (MA-Fungi 88096), Lado 23434 (MA-Fungi 88280). PER-14-17: Armatocereus sp. remains, Lado 23503 (MA-Fungi 88297), Lado 23504 (MA-Fungi 88298); twigs, Lado 23505 (MA-Fungi 88299). PER-14-18: bark, Lado 23510 (MA-Fungi 88305).

Widely distributed throughout the Neotropics. In Peru previously reported from Loreto (Wrigley de Basanta \& al., 2008a). Collection aet 12335 b has been left as cf. since the spores were irregular, probably due to incomplete development, although the other characters were typical.

\section{*Physarum spectabile Nann.-Bremek., Lado \& G. Moreno Figs. 53-56.}

PER-12-26: aerial litter (mc, pH 5.42), sls 30381. PER-12-42: Alternantera pubiflora twigs and leaves, Lado 22027 (MA-Fungi 87820). PER-12 47: Tillandsia purpurea leaf bases (mc, $\mathrm{pH}$ 7.43), dwb 3566. PER-14-11: Capparis scabrida leaf litter (mc, pH 7.84), dwb 3718.

In South America, only previously known from Chile (Lado \& al., 2007, 2013) and Argentina (Lado \& al., 2011, 2014). This species usually develops on cacti and succulents but was found in Peru on plants that stabilise the dunes. The characteristic dark, angular spores ornamented with prominent spines and the calcareous nodes of the capillitium are illustrated by SEM in Figs. 53-56.

\section{*Physarum straminipes Lister}

PER-12-43: twigs, Lado 22079 (MA-Fungi 87865). PER-13-29: Capparis scabrida twigs and leaves, Lado 22617 (MA-Fungi 87996).

In South America, only previously known from the Monte Desert and Patagonia in Argentina (Lado \& al., 2011, 2014), and the Atacama Desert and dry regions of Chile (Lado \& al., 2007, 2013).

\section{Physarum viride (Bull.) Pers.}

PER-13-27: twigs and litter under Schinus molle, Lado 22605 (MA-Fungi 87983). 
Widely distributed throughout the Neotropics. In Peru only reported from Loreto (Stephenson \& Mitchell, 1994).

\section{*Stemonaria gracilis Nann.-Bremek. \& Y. Yamam. Figs. 57-69.}

PER-12-26: ground litter ( $\mathrm{mc}, \mathrm{pH}$ 4.59), sls 29156. PER-12-28: ground litter (mc, $\mathrm{pH}$ 6.38), sls 28354. PER-12-34: Tillandsia latifolia inflorescence (mc, pH 6.3), aet 12274 (MA-Fungi 88437), (mc, pH 5.4), aet 12341, (mc, $\mathrm{pH} 7.2$ ), aet 12352; mixed Tillandsia (mc, $\mathrm{pH} 5.68$ ), sls 28442 (MA-Fungi 88446); mixed Tillandsia (mc, pH 5.71), sls 28850. PER-12-47: Tillandsia purpurea leaf bases (mc, pH 7.43), dwb 3539 (mc, pH 7.43), dwb 3544; Tillandsia purpurea leaf bases (mc, pH 5.18), dwb 3558 (mc, pH 5.18), dwb 3565. PER-14-19: Agave americana leaves, Lado 23518 (MA-Fungi 88313).

Reported previously from Loreto, Peru by Wrigley de Basanta \& al. (2008) on lianas. Otherwise only reported in the Neotropics from Costa Rica by Rojas \& al. (2010). This is a rare species that appeared frequently in the moist chamber cultures of Tillandsia remains. The sporocarp, the stalk and details of the capillitial net are illustrated for the first time by SEM (Figs. 57-61). The stalk is clearly formed of concentric layers making a tube (Figs. 60-61), not of fibers. The spores by LM are defined as warted-reticulate, by SEM (Figs. 62-69) they show a well defined net and a complex ornamentation of warts connected in rows in a pattern of a "simple reticulate type with perforated muri" according to the terminology of Rammeloo (1975), and, in some cases, the pillars supporting the muri surpass the top of the muri (Fig. 67), while in other the muri appear more solid (Figs. 68-69).

\section{*Stemonitis herbatica Peck}

PER-14-18: wood, Lado 23508 (MA-Fungi 88302).

Distributed throughout the Neotropics, but not previously reported from Peru.

\section{Stemonitis splendens Rostaf.}

PER-14-34: wood, Lado 23559 (MA-Fungi 88347).

Widely distributed throughout the Neotropics. In Peru only reported from Junín (Zúñiga de Acleto, 2010) and Loreto (Stephenson \& Mitchell, 1994).

\section{${ }^{\circ}$ Stemonitopsis gracilis (G. Lister) Nann.-Bremek.} Figs. 43-44.

PER-14-12: Neoraimondia sp. remains, Lado 23429 (MA-Fungi 88093).

Not previously reported from South America. Only one collection of grouped and dispersed but well developed sporocarps, up to $2 \mathrm{~mm}$ tall, and cylindrical. The capillitial threads are undulate, without membranous expansions, forming a broken surface net. The spores are pale brown by TL, with a paler area corresponding to the area of germination, and minutely warted, these characters agree with the description of Nannenga-Bremekamp (1975). However, the spores in the Peruvian specimens are larger, 7-9(-10) $\mu \mathrm{m}$ diam vs $5-7 \mu \mathrm{m}$ diam as described by Nannenga-Bremekamp (1975), and by SEM they are densely warted with some of the warts fused or connected by a tenuous basal "muri" (Figs. 43-44).

Stemonitopsis typhina (F.H. Wigg.) Nann.-Bremek. PER-14-34: wood, Lado 23558 (MA-Fungi 88346).

Distributed throughout the Neotropics. In Peru only previously known from a record by Farr (1976) preserved in the BPI as Comatricha typhoides (Bull.) Rostaf.

*Trichia agaves (G. Moreno, Lizárraga \& Illana)
Mosquera, Lado, Estrada \& Beltrán-Tej.

PER-14-19: Agave americana leaves, Lado 23519 (MA-Fungi 88314), Lado 23520 (MA-Fungi 88315), Lado 23521 (MA-Fungi 88316).

In South America previously reported only from Brazil (Cavalcanti, 2010). The three collectios were obtained on the same typical substrate of the species, Agave americana, and the characters agree with the descriptions available in the literature.

Willkommlangea reticulata (Alb. \& Schwein.) Kuntze

PER-14-06: Prosopis sp. bark (mc, pH 4.81), dwb 3728.

Widely distributed throughout the Neotropics. In Peru only reported from Loreto (Wrigley de Basanta \& al., 2008a).

\section{DISCUSSION}

As stated above, the results of the survey of these hyperarid habitats yielded 723 collections of myxomycetes representing 78 species in 23 genera in all six orders recognised in this group. These include one species that is new to science, another 2 species that have not been reported before from the Neotropics, a further 4 new records for South America and 41 additional species reported for the first time in Peru. This increases the number of myxomycetes recorded from the country so far by over $50 \%$, to 128 species.

The known distribution of many species has been extended by these results, which also fill a prominent gap in the knowledge of these microorganisms in a virtually unexplored biogeographical area. These data complement work carried out in other South American deserts such as the Atacama, Chile (Lado \& al., 2007, 2013) and Monte, Argentina (Lado \& al., 2011) and help to develop an idea of the true myxobiota of the Neotropics. It is becoming increasingly evident that both warm and cold deserts are reservoirs of surprising myxomycete biodiversity, as results from the Patagonian steppe (Lado \& al., 2014) and work in other arid areas of Europe and Asia (Schnittler, 2001; Novozhilov \& al., 2006; Schnittler \& al., 2013) also indicate.

The predominant order among the results was the Physarales with 44 species, $56 \%$ of the recovered taxa. This was followed by the Trichiales, with 14 species, and the Stemonitales, with 10 species. The orders with the lowest number of species were the Liceales (5 species), Echinosteliales (4 species) and Ceratiomyxales with only one species. The predominance of the Physarales in arid zones in the Americas has previously been documented by surveys carried out in the Valle de Tehuacán-Cuicatlán, 



Figs. 57-69. SEM images of Stemonaria gracilis. 57. Sporocarp (Lado 23518). 58. Detail of the columella and capillitium (Lado 23518). 59. Detail of the peripheral capillitium (Lado 23518). 60. Base of the stalk (aet 12274). 61. Base of the stalk (sls 28442). 62. Group of spores (Lado 23518). 63-64. Spores (Lado 23518). 65. Spore (aet 12274). 66. Spore (sls 28442). 67. Detail of the ornamentation of the spore (Lado 23518). 68. Detail of the ornamentation of the spore (aet 12274). 69. Detail of the ornamentation of the spore (sls 28442). Bar: $57=1 \mathrm{~mm} ; 58=100 \mu \mathrm{m} ; 59,62-66=10 \mu \mathrm{m}$; $60=20 \mu \mathrm{m} ; 61=50 \mu \mathrm{m} ; 67-69=1 \mu \mathrm{m}$.

Mexico (Estrada-Torres \& al., 2009) and Monte Desert, Argentina (Lado \& al., 2011), and has also been registered in the cold Central Asian deserts in Kazakhstan, Mongolia and Caspian lowland by Schnittler (2001) and Novozhilov \& al. (2006). The prevalence of this order is even more obvious when the total number of collections is taken into consideration. Almost $79 \%$ (566) of the collections belonged to this order, $14 \%$ to the Trichiales and only $7 \%$ to the remaining four orders. The most commonly collected species were Badhamia melanospora (139 collections), Physarum atacamense (135), Didymium nigrisporum (65), Physarum pusillum (47), Physarum clavisporum (35), Perichaena vermicularis (33), Arcyria insignis (26) and Physarum compressum (21). The four first species can be 
considered to be particulary abundant ( $>6 \%$ relative abundance) and the most representative of this Peruvian coastal desert since they make up $53.5 \%$ of all collections. At the other extreme $41 \%$ of the species studied were represented by only a single collection.

Of the 78 species identified from this survey, 33 were only collected in the field and 20 were obtained only from moist chamber culture while 25 were recovered from both. In the field collections, $70 \%$ of the specimens found were represented by 5 species, whereas in moist chamber cultures this percentage was made up of 11 species. The six additional species, abundant in moist chamber culture were rarely collected in the field or not at all. Both techniques returned the same three most dominant species. The two methods of survey are evidently complementary to each other and give a more accurate account of the myxobiota actually present in an area, especially in arid places like the study area where environmental extremes can affect the detection of many species in the field. This is reinforced by the tests of independence done on the proportion of collections (Table 2 ) and species (Table 3 ) recovered from the field or from moist chamber culture. For the proportion of collections from different orders $\mathrm{X}^{2}=68.63(\mathrm{df}=2$; $\mathrm{p}<0.0001$ ), indicating that the number of collections of different groups of myxomycetes depends on whether they were collected in the field or from moist chamber culture. For the proportion of species from different orders, however, $X^{2}=1.32(d f=2 ; p=0.5177)$, which indicates that this is independent of the method used to collect the species (field or culture).

The fact that the Physarales were also the predominant order considering only the field collections, since the five most common species were from this order, could be due to various factors. One of these could be that this group may have strategies to complete their development, even under such extreme conditions, that the other orders of myxomycetes do not possess. It is also possible that the reproductive structures of the Physarales are more resistant to destruction by biotic and abiotic elements in arid zones and so remain for longer periods in situ. The first of these hypotheses is supported in this study by the fact that even in moist chamber culture, where available moisture is a constant, the Physarales were the dominant group, and in addition the moisture and favourable conditions permitted the development of a larger number of specimens from other orders, the Echinosteliales, Liceales and Stemonitales. In

Table 2. Comparison of sampling methods for collections.

\begin{tabular}{lccc}
\hline & Physarales & Trichiales & Other orders \\
\hline Field collected & 424 & 65 & 11 \\
$\begin{array}{l}\text { Isolated from moist } \\
\text { chamber culture }\end{array}$ & 142 & 37 & 42 \\
\hline
\end{tabular}

Table 3. Comparison of sampling methods for species.

\begin{tabular}{lccc}
\hline & Physarales & Trichiales & Other orders \\
\hline Field collected & 36 & 11 & 11 \\
$\begin{array}{l}\text { Isolated from moist } \\
\text { chamber culture }\end{array}$ & 23 & 10 & 12 \\
\hline
\end{tabular}

the field, under natural conditions, species from the latter orders would be opportunistic, developing only when sporadic moisture was available in these dry environments of the Peruvian coast.

The most productive substrates were leaves and other dead parts of species of the genus Capparis with 158 collections from 14 myxomycete species. The species of this plant genus, as noted above, stabilise the dunes in the coastal desert and were particularly common in the more northerly areas (Fig. 7). The species of Tillandsia, found sometimes as isolated islands of vegetation sprouting directly out of the sand dunes (Figs. 9-10), produced 86 collections of myxomycete $(12 \%)$ but these represented 20 species, and the remains of species of Haageocereus were the third most productive substrate genus with 57 collections. However if the remains of all the species of cacti are taken together they accounted for $23 \%$ (167) of the collections but representing only 15 species of myxomycete, and so accounting for considerably less diversity than the Tillandsias. The locality that produced the greatest number of myxomycetes with 65 collections was Yauyos (PER-12-34) in the administrative department of Lima at $13^{\circ} \mathrm{S}$ latitude and an elevation of $400 \mathrm{~m}$. The greatest variety (number of species) was collected from Pariacoto in Ancash, $9^{\circ} \mathrm{S}$ latitude and at $1478 \mathrm{~m}$ (locality PER-13-32) where 18 different species were recorded. More than half the collections were produced by only 14 localities (375 collections). The localities from South latitude $11^{\circ}$ to South latitude $17^{\circ} \mathrm{had}$ a greater number of collections than South latitudes $3^{\circ}$ to $9^{\circ}$, when corrected for the number of collecting localities sampled, which is surprising, given the proximity of the latter to the tropical areas and the subsequent increased variety in vegetation. However the elevations where the greatest number of collections were made were between 400 and $600 \mathrm{~m}$, when corrected for the number of localities sampled at each elevation. This suggests a reason for the number and variety of myxomycetes at the above localities, since at these latitudes and elevations the coastal fogs or garuas constantly bathe the slopes providing more moisture for the development of these microorganisms (Figs. 13-14). This moisture is responsible for the great variety of plants now known from the lomas formations in these areas, known by some authors as "fog oases"(Dillon, 1997). These pockets of vegetation enable the myxomycetes to exploit the multiple microhabitats available to complete their life cycles in such an otherwise hostile environment of barren desert, and are most important areas of conservation.

The substrate $\mathrm{pH}$ in moist chamber cultures has been shown to influence the number and variety of myxomycetes isolated from a particular substrate (Wrigley de Basanta, 2004). In these cultures the range of substrate $\mathrm{pH}$ of the 225 moist chamber collections was from 4.39 to 8.13 , but more than half the collections were obtained from cultures with circumneutral $\mathrm{pH}$ (Fig. 70). This has been found in other surveys (Wrigley de Basanta \& al., 2008a; Estrada Torres $\&$ al., 2009; Lado \& al., 2011).

According to the estimators CHAO1 and ACE, the expected species richness if the sampling effort had been exhaustive would be 119 and 114 respectively (Fig. 71). The estimated number of species based on the Clench function, with an $\mathrm{r}^{2}=0.996$, was 124 , indicating a good fit of the 


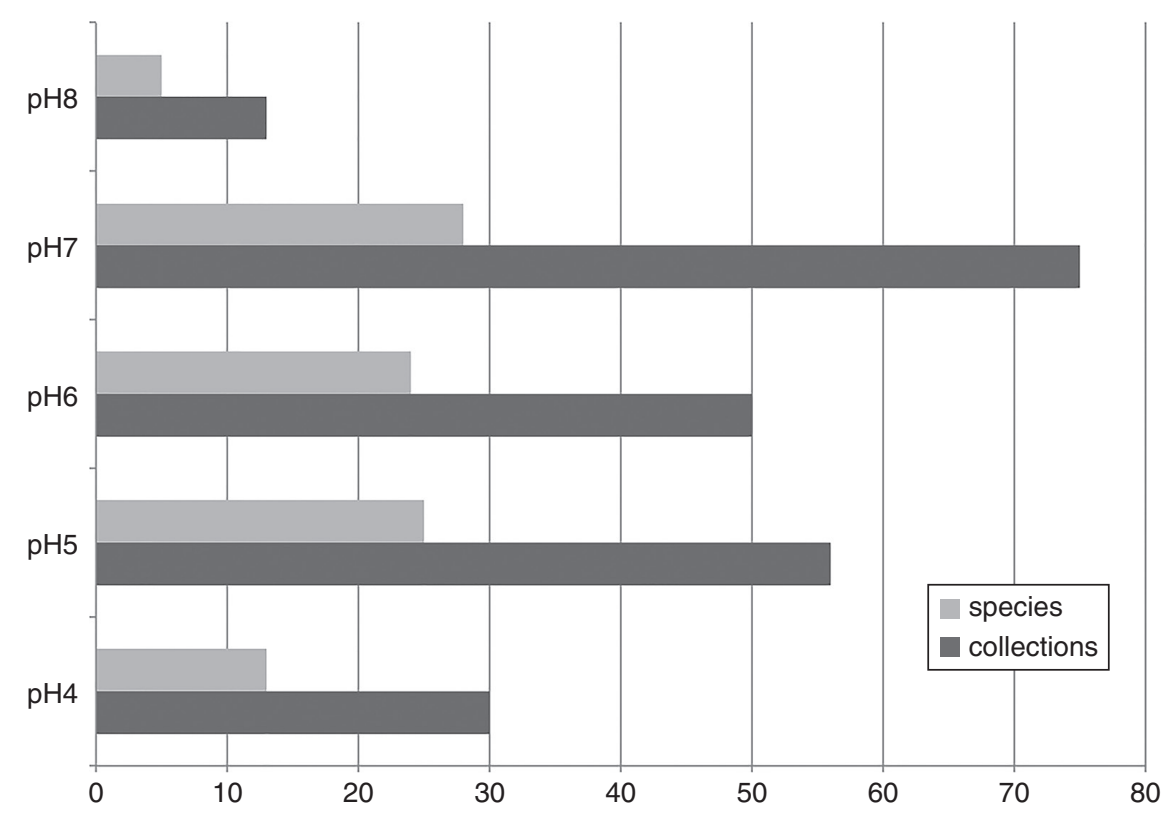

Fig. 70. Number of collections and species of myxomycete at each different substrate $\mathrm{pH}$.

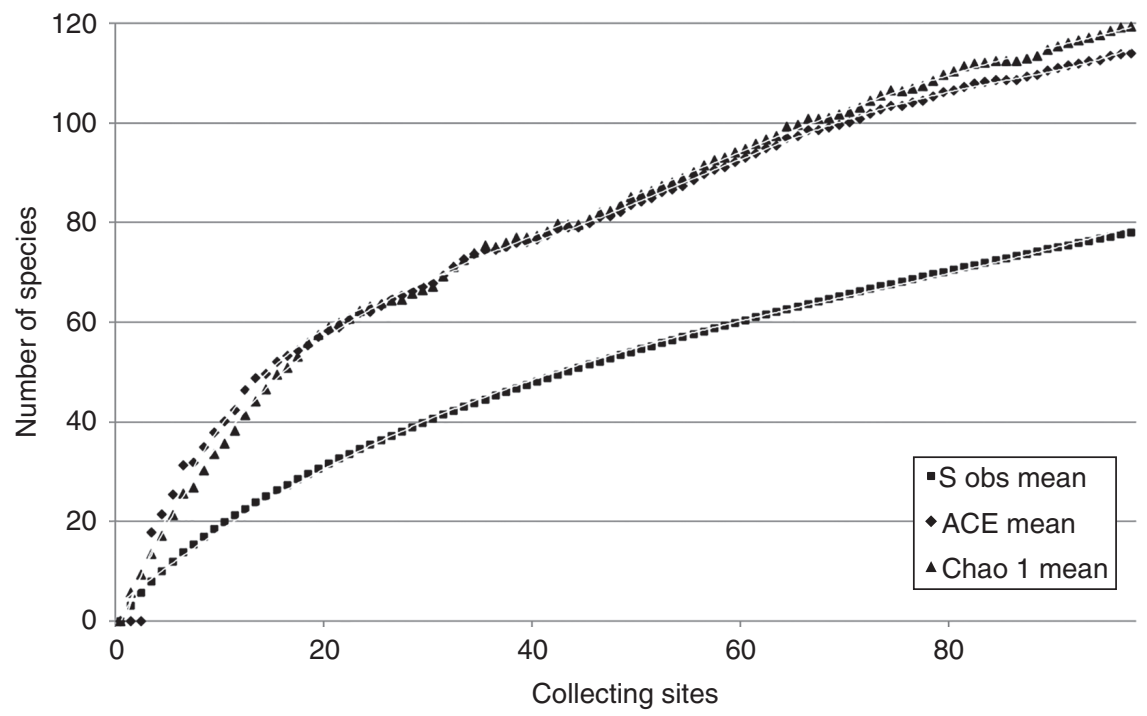

Fig. 71. Species accumulation curves using ACE and CHAO1.

data to the curve (Fig. 72) and giving a value slightly higher than that estimated using CHAO 1. This means that in this survey almost $70 \%(63 \%-69 \%)$ of all possible species in the sampling area were recorded, a very representative result considering the extreme conditions of the coastal desert. Extrapolation of the species richness using the program EstimateS showed that if 49 more collecting localities had been visited this would have returned only 17 species more. The Clench function suggests that 102 more collecting sites would be necessary to achieve these 17 more species, with the enormous effort in both the field and laboratory work implied. In fact double the time of 3 weeks a year for three years invested in sampling, and the consequent 3 years of laboratory work, would be required. This is because of the large number of rare species among the myxobiota of this region.
Analysis of the results of field collections alone using the above estimators showed that an approximate $63 \%$ of the species theoretically possible were recorded. Both CHAO1 and ACE showed that $66 \%$ and $73 \%$ of possible species were recovered using moist chamber cultures, a slightly more efficient result probably on account of the more stable favourable conditions that existed in the culture. This productivity is similar to that of other surveys (Lado \& al., 2011, $2013,2014)$ and surprisingly high considering the arid environment and the multiple reasons why all the myxomycetes actually present do not fruit normally and produce identifiable specimens. The exact phenological optimum varies among species and will not always coincide with the moment of sampling in the field. In culture many sclerotia and probably microcysts never developed further, and some abnormal fruitings were found that were impossible to identify. 


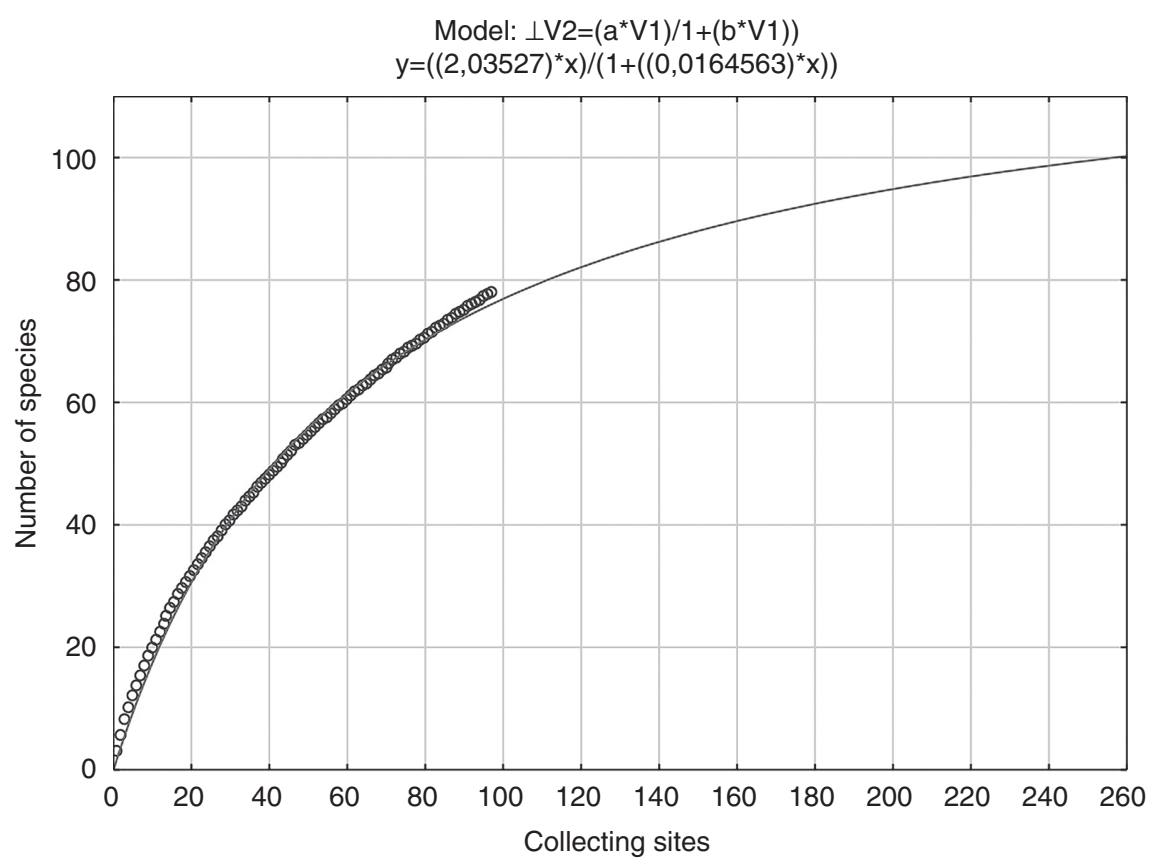

Fig. 72. Species accumulation curve using the adjustment by Clench.

The results are also surprising, given the vastness of the area studied, the isolation of many of the groups of plants that act as substrates for the development of these organisms, in pockets or islands, and the fact that even in spite of these factors, from $86 \%$ of the localities visited identifiable myxomycetes were recovered.

The biotic distinctness, or complementarity, of the assemblage of myxomycetes in the study area compared to that of other arid areas in South America is shown in Table 4.

Not surprisingly the lowest values, or most similar communities are the closest arid areas in North Chile and Argentina. All three belong to the South American Transition Zone, according to Morrone (2004). However, in both cases there is an approximate $70 \%$ difference in the species present, explainable by the peculiarity of the myxobiota of the Peruvian coast. Rarer species such as Didymium nigrisporum, Didymium anomalum, Didymium cf. rugulosporum, Licea bulbosa and Stemonaria gracilis are unique to the Peruvian biota, as is the newly described species, Didymium peruvianum. Some species are even shared with those found in North American arid areas such as Diderma acanthosporum and Perichaena luteola (Estrada-Torres \& al., 2009). However the shared species between the three South American deserts include Echinostelium arboreum, Licea succulenticola or Physarum licheniforme (Lado \& al., 2011, 2013), and species hitherto known only from South American deserts such as Licea eremophila and Physarum atacamense (Wrigley de
Basanta \& al., 2010a; 2012). The striking difference between the species found in Central Chile and the study area, where $87 \%$ of the species are different, could be due to the clearly Mediterranean vegetation of the former favouring the development of foliicolous species such as Didymium chilense Estrada, Lado \& D. Wrigley, Didymium comatum (Lister) Nann.-Bremek., Didymium eximium Peck, Didymium laxifilum G. Lister \& J. Ross and Physarum newtonii T. Macbr. (Lado \& al., 2013), that were not present in the coastal desert of Peru. The Patagonian steppe and southern Chile are areas with almost $80 \%$ of species distinct from the coastal desert of Peru, reflecting the geographical distance and marked ecological differences between the two regions. It is perhaps important to point out that the moderately high and high complementarity between the coastal desert of Peru and other South American arid areas reinforces once again the idea that each biogeographical area has its own characteristic myxobiota, as has already been pointed out in other American studies (Estrada-Torres \& al., 2009; Wrigley de Basanta \& al., 2010b; Lado \& al., 2011, 2013). These patterns of distribution may also result from the geographic barrier formed by the Andes and the corridors of the interandean valleys that permit interchange of some but not all biota.

In summary, the present paper represents the first systematic study of the myxomycetes from this arid biogeographical area, previously virtually unexplored for these microorganisms. The results obtained support the fact that

Table 4. Percentage complementarity of the assemblage of myxomycetes from the Peruvian coastal desert with other arid areas in South America.

\begin{tabular}{llllll}
\hline & Monte desert Argentina & Atacama desert Chile & Central Chile & South Chile & \multicolumn{1}{c}{ Patagonia } \\
\hline References & Lado \& al. (2011) & Lado \& al. (2007, 2013) & Lado \& al. (2013) & Lado \& al. (2013) & Lado \& al. (2014) \\
Peru & 0.696 & 0.717 & 0.867 & 0.787 & 0.779 \\
\hline
\end{tabular}


the coastal desert of Peru is surprisingly rich in myxomycetes, from each taxonomic order of the group, and that even within South America, each arid area has a unique assemblage of the organisms, although there are species common to all. The results also indicate that the myxomycetes are a normal component of the Peruvian desert flora, with an ecological role that is yet to be fully determined, since they have been isolated from the typical vegetation of the area including endemic plants. Although cacti, as is customary in drylands of the Americas, were important substrates with a high yield of myxomycete collections, the two plant genera that proved to be the most productive were the genus Capparis and the genus Tillandsia. These are important components of the lomas formations, essential for stabilising the sand dunes, and appear to be a natural habitat for myxomycetes. These were even more abundant and varied in areas under the influence of coastal fogs. In spite of the vast and varied distances between the lomas formations that make up much of the vegetation in this study, and the extreme aridity of some places, myxomycetes were found in $87 \%$ of the locations sampled. This raises questions as to the methods of dispersal and distribution of the organisms over such disjunct geographically isolated formations, designated unique delimited ecosystems by Dillon \& al., (2011). These data not only refute the paradigm that the myxomycetes are associated mainly with humid temperate and tropical ecosystems but also suggest that these microorganisms are important elements of biodiversity and should certainly be considered in establishing conservation priorities in these fragile ecosystems.

\section{ACKNOWLEDGMENTS}

This research was supported by the Spanish Government grants CGL2011-22684 and CGL2014-52584P. We are grateful to Asuncion Cano, Museo de Historia Natural, Lima, Peru, and Blanca León, Universidad Nacional Mayor de San Marcos, Lima, Peru, for their assistance with collecting permits, and other aspects of the project, and to Adam Rollins, Gloria Rebaza, Italo Treviño, Joaquina García, Jenny Rojas-Fox, Johanny Molina and Susi Castillo for their help with fieldwork. We also thank Yolanda Ruiz for her technical assistance with SEM, Carlos de Mier for his help with the images and micrographs, and Juan Carlos Hernández for database management.

\section{REFERENCES}

Bezerra, A.C.C., de Lima, V.X., Tenorio, J.C.G. \& Cavalcanti, L.H. 2014 Myxomycetes in Alagoas state (Brazil) and notes on its distribution. Biotemas 27(3): 13-22. http://dx.doi.org/10.5007/2175-7925. $2014 \mathrm{v} 27 \mathrm{n} 3 \mathrm{p} 13$

Camino, M., Stephenson, S.L., Krivomaz, T., Wrigley de Basanta, D., Lado, C. \& Estrada-Torres, A. 2008. Biodiversity survey for myxomycetes in the mountains of central Cuba. Revista Mexicana de Micología 27:39-51.

Cavalcanti, L.H. 2002. Biodiversidade e distribuição de mixomicetos em ambientes naturais e antropogênicos no Brasil: espécies ocorrentes nas Regiões Norte e Nordeste. In: Araújo, E.L. \& al. (eds.). Biodiversidade, Conservaçao e Uso Sustentável da Flora do Brasil. UFPE. Sociedade Botânica do Brasil, Recife, pp. 209-216.

Cavalcanti, L.H. 2010. Stemonitales. In: Forzza \& al. (eds.). Catálogo de plantas e fungos do Brasil. 1: 100-102. Rio de Janeiro.

Clark, J.D. \& Landolt, J.C. 2001. Myxomycete biosystematics: various Didymium and Physarum species. Nova Hedwigia 73(3-4): 437-444.

Colwell, R.K. 2013. EstimateS, version 9.1: Statistical Estimation of Species Richness and Shared Species from Samples. Available from http://viceroy.eeb.uconn.edu/estimates/

Colwell, R.K. \& Coddington. J.A. 1994. Estimating terrestrial biodiversity through extrapolation. Philosophical Transactions of the Royal Society, Series B 345: 101-118. http://dx.doi.org/10.1098/rstb.1994.0091
Colwell, R.K., Mao, C.X. \& Chang, J. 2004. Interpolating, extrapolating, and comparing incidence-based species accumulation curves. Ecology 85: 2717-2727. http://dx.doi.org/10.1890/03-0557

Dillon, M.O. 1997. Lomas formations Peru. In: Davis, S.D. \& al. (eds.) Centres of plant diversity. Vol. 3. The Americas. IUCN Publication Unit. Cambridge, U.K., pp. 519-527.

Dillon, M.O., Leiva González, S., Zapata Cruz, M., Lezama Asencio, P. \& Quipuscoa Silvestre, V. 2011. Floristic Checklist of the Peruvian Lomas Formations. Catálogo florístico de las Lomas peruanas. Arnaldoa 18(1): 7-32.

Esqueda, M., Lizárraga, M., Gutiérrez, A., Coronado, M.L., Valenzuela, R., Raymundo, T., Chacón, S., Vargas, G. \& Barredo-Pool, F. 2011. Diversidad fúngica en planicies del desierto central sonorense y centro del desierto chibuabuense. Informe final SNIB-CONABIO Proyecto GT016, México, D.F.

Estrada-Torres, A., Lado, C. \& Rodríguez-Palma, M. 2001. Two new species of Myxomycetes from a tropical deciduous forest of Mexico. Mycologia 93(4): 744-750. http://dx.doi.org/10.2307/3761829

Estrada-Torres, A., Wrigley de Basanta, D., Conde, E. \& Lado, C. 2009. Myxomycetes associated with dryland ecosystems of the TehuacánCuicatlán Valley Biosphere Reserve, Mexico. Fungal Diversity 36: 17-56.

Farr, M.L. 1968. An illustrated key to the Myxomycetes of South America, with special reference to Brasil. Rickia 3: 45-88.

Farr, M.L. 1976. Myxomycetes. Flora Neotropica 16: 1-305.

Gómez-Sosa, E. 1986. Astragalus neobarnebyanus (Leguminosae): A New Species from Peru. Brittonia 38(4): 427- 429. http://dx.doi.org/10.2307/ 2807094

Gottsberger, G. 1968. Myxomycetes aus Bahia und Goiás. Nova Hedwigia 15:361-368.

Henney, M.R., Alexopoulos, C.J. \& Scheetz, R.W. 1980. Didymium atrichum, a new Myxomycete from South-Central Texas. Mycotaxon 11(1): 150-164.

Jiménez-Valverde J. \& Hortal, J. 2003. Las curvas de acumulación de especies y la necesidad de evaluar la calidad de los inventarios biológicos. Revista Ibérica de Aracnología 8: 151-161.

Keller, H.W. 1970. Didymium saturnus, a new Myxomycete occurring on straw stacks. Mycologia 62(5): 1061-1066. http://dx.doi.org/10.2307/ 3757619

Kirk, P.M., Cannon, P.F., Minter, D.W. \& Stalpers, J.A. 2011. Dictionary of the Fungi. CABI International. Oxon, UK.

Kowalski, D.T. 1969. A new coprophillous species of Didymium. Mycologia 61(3): 635-639. http://dx.doi.org/10.2307/3757253

Kowalski, D.T. 1972. Squamuloderma: a new genus of Myxomycetes. Mycologia 64(6): 1282-1289. http://dx.doi.org/10.2307/3757965

Lado, C. 2005-2015. Nomen.eumycetozoa.com. An online nomenclatural information system of Eumycetozoa. Real Jardín Botánico, CSIC. Madrid, Spain. Available from: http://www.nomen.eumycetozoa.com (accessed: 10 July 2015).

Lado, C., Estrada-Torres, A. \& Stephenson, S.L. 2007. Myxomycetes collected in the first phase of a north-south transect of Chile. Fungal Diversity 25: 81-101.

Lado, C., Mosquera, J. \& Beltrán-Tejera, E. 1999. Cribraria zonatispora, development of a new myxomycete with unique spores. Mycologia 91(1): 157-165. http://dx.doi.org/10.2307/3761205

Lado, C., Mosquera, J., Estrada-Torres, A. \& Wrigley de Basanata, D. 2007. Description and culture of a new succulenticolous Didymium (Myxomycetes). Mycologia 99(4): 602-611. http://dx.doi.org/10.3852/ mycologia.99.4.602

Lado, C. \& Wrigley de Basanta, D. 2008. A review of Neotropical Myxomycetes 1828-2008). Anales del Jardín Botánico de Madrid 65(2): 211-254. http://dx.doi.org/10.3989/ajbm.2008.v65.i2.293

Lado, C., Wrigley de Basanta, D. \& Estrada-Torres, A. 2011. Biodiversity of Myxomycetes from the Monte Desert of Argentina. Anales del Jardín Botánico de Madrid 68(1): 61-95. http://dx.doi.org/10.3989/ ajbm.2266

Lado, C., Wrigley de Basanta, D., Estrada-Torres, A., García Carvajal, E., Aguilar, M. \& Hernández, J.C. 2009. Description of a new species of Perichaena (Myxomycetes) from arid areas of Argentina. Anales del Jardín Botánico de Madrid 66(1): 63-70.

Lado, C., Wrigley de Basanta, D., Estrada-Torres, A. \& García-Carvajal, E. 2014. Myxomycete diversity of the Patagonian Steppe and bordering areas in Argentina. Anales del Jardín Botánico de Madrid 71: e006 (1-35) http://dx.doi.org/10.3989/ajbm.2394

Lado, C., Wrigley de Basanta, D., Estrada-Torres, A. \& Stephenson, S.L. 2013. The biodiversity of myxomycetes in central Chile. Fungal Diversity 59: 3-32. http://dx.doi.org/10.1007/s13225-012-0159-8 
Leiva González, S., Zapata Cruz, M., Gayoso Bazán, G., Chang Chávez, L., Dillon, M.O. \& Quipuscoa Silvestre, V. 2014. Diversidad Florística de la Loma Cerro Campana, Provincia Trujillo, Departamento La LibertadPerú. Arnaldoa 21(1): 187-220.

Lister, G. 1931. New species of Mycetozoa from Japan. Journal of Botany 69: 297-298

Lizárraga, M., Moreno, G., Esqueda, M., Salazar-Márquez, C. \& Coronado, M.L. 2015. Myxomycetes of Sonora (Mexico) 6. Central plains of the Sonoran Desert. Mycotaxon 130: 145-164. http://dx.doi.org/10 5248/130.145

Martin, G.W. \& Alexopoulos, C.J. 1969. The Myxomycetes. Univ. Iowa Press. Iowa

McHugh, R. 2005. Moist Chamber Culture and Field Collections of Myxomycetes from Ecuador. Mycotaxon 91: 107-118.

McHugh, R. 2009. Field and moist chamber collections of Paraguay myxomycetes. Karstenia 48: 49-56.

Moreno, G., Lizárraga, M. \& Illana, C. 1997. A rare Didymium from Mexico (Myxomycetes). Cryptogamie Mycologie 18(4): 327-331.

Moreno, G., Lizárraga, M. \& Illana, C. 2007. Catálogo de los Myxomycetes de México. Boletín de la Sociedad Micológica de Madrid 31: 187-229.

Moreno, G., Castillo, A., Deschamps, J.R. \& Hladki, A.I. 2012. Critical revision of some Myxomycetes kept at the Buenos Aires BAFC Herbarium and Tucumán LIL Herbarium. II. Boletín de la Sociedad Micológica de Madrid 36: 81-92.

Morrone, J.J. 2004. La Zona de Transición Sudamericana: Caracterización y relevancia evolutiva. Acta Entomológica Chilena 28: 41-50.

Mosquera, J., Lado, C., Estrada-Torres, A., Beltrán-Tejera, E. \& Wrigley de Basanta, D. 2003. Description and Culture of a new Myxomycete, Licea succulenticola. Anales del Jardín Botánico de Madrid 60(1): 3-10

Nannenga-Bremekamp, N.E. 1966. Notes on Myxomycetes XI. Some new species of Stemonitis, Comatricha, Badhamia, Physarum, Diderma and Didymium. Proceedings Konienklijke Nederlandse Akademie van Wetenschappen Series C 69(3): 350-363.

Nannenga-Bremekamp, N.E. 1975. De Nederlandse Myxomyceten. Bibliotheek van de Koninklijke Nederlandse Natuurbistorische Vereniging 18: 1-440.

Nannenga-Bremekamp, N.E. 1989. Notes on myxomycetes XXIII Proceedings Konienklijke Nederlandse Akademie van Wetenschappen Series C 92(4): 505-515.

Nannenga-Bremekamp, N.E. 1991. A guide to temperate Myxomycetes Biopress Limited. Bristol.

Nannenga-Bremekamp, N.E., Mukerji, K.G. \& Pasricha, R. 1984. Notes on Indian Myxomycetes. Three new species, and comments on others Proceedings Konienklijke Nederlandse Akademie van Wetenschappen Series C 87(4): 471-482.

Neubert, H., Nowotny, W. \& Baumann, K. 1995. Die Myxomyceten Deutschland und des angrenzenden Alpenraumes unter besonderer Berücksichtigung Österreichs. Band 2. Physarales. Karlheinz Baumann Verlag, Gomaringen.

Novozhilov, Y.K., Zemlyanskaya, I.V., Schnittler, M. \& Stephenson, S.L. 2006. Myxomycete diversity and ecology in the arid regions of the Lower Volga River Basin (Russia). Fungal Diversity 23: 193-241.

Oltra, M. 2003. Contribución al conocimiento de los Myxomycetes de la provincia de Valencia (España). VI. Butlletí Societat Micològica Valenciana 7: 3-34.

Patouillard, N. \& Lagerheim, G. de 1895. Champignons de l'Equateur (Pugillus V). Bulletin de la Société Mycologique de France 11: 205-234.

Poulain, M., Meyer, M. \& Bozonnet, J. 2011. Les Myxomycètes. Tome 1. Guide de détermination. Fédération mycologique et botanique DauphinéSavoie. Sevrier.

Rammeloo, J. 1975. Structure of the epispore in the Stemonitales (Myxomycetes) as seen with the scanning electron microscope. Bulletin del Jarin. Botanique National de Belgique 45: 301-306. http://dx.doi. org/10.2307/3667483

Rojas, C., Morales, R.E., Calderón I. \& Clerc, P. 2013. First records of myxomycetes from El Salvador. Mycosphere 4(6): 1042-1051.

Rojas, C., Schnittler, M., Biffi, D. \& Stephenson, S.L. 2008. Microhabitat and niche separation in species of Ceratiomyxa. Mycologia 100(6): 843-850. http://dx.doi.org/10.3852/07-197

Rojas, C., Schnittler, M. \& Stephenson, S.L. 2010. A review of the Costa Rican myxomycetes (Amebozoa). Brenesia 73-74:39-57.
Rojas, C., Stephenson, S.L. \& Pavlich, M. 2011. New additions to the myxobiota of Peru. Mycosphere 2(5): 583-592. http://dx.doi.org/10.5943/ mycosphere $/ 2 / 5 / 8$

Rossman, A.Y., Tulloss, R.E., O'Dell, T.E. \& Thorn, R.G. 1998. Protocols for an all taxa biodiversity inventory of Fungi in a costa rican conservation area. Parkway Oublishers, Inc. Boone, North Carolina.

Rudolphi, F. 1829. Plantarum vel novarum vel minus cognitarum descriptiones. Linnaea 4: 114-120.

Rundel, P.W., Dillon, M.O., Palma, B., Mooney, H.A., Gulmon, S.L. \& Ehleringer, J.R. 1991. The Phytogeography and Ecology of the Coastal Atacama and Peruvian Deserts. Aliso 13(1): 1-49.

Salazar-Márquez, C., Lizárraga, M. \& Moreno, G. 2014. Myxomycetes de matorral xerófilo del municipio de Juárez, Chihuahua, México. Boletín de la Sociedad Micológica de Madrid 38: 67-77.

Schnittler, M. 2001. Ecology of myxomycetes of a winter-cold desert in western Kazakhstan. Mycologia 93(4): 653-669. http://dx.doi.org/ $10.2307 / 3761820$

Schnittler, M. \& Stephenson, S.L. 2002. Inflorescences of Neotropical herbs as a newly discovered microhabitat for myxomycetes. Mycologia 94(1): 6-20. http://dx.doi.org/10.2307/3761841

Schnittler, M., Lado, C. \& Stephenson, S.L. 2002. Rapid biodiversity assessment of a tropical myxomycete assemblage-Maquipucuna Cloud Forest Reserve, Ecuador. Fungal Diversity 9: 135-167.

Schnittler, M., Novozhilov, Y.K., Carvajal, E. \& Spiegel, F.W. 2013. Myxomycete diversity in the Tarim basin and eastern Tian-Shan, Xinjiang Prov., China. Fungal Diversity 59: 91-108. http://dx.doi.org/10.1007/ s13225-012-0186-5

Stephenson, S.L. 1989. Distribution and eology of myxomycetes in temperate forests. II. Patterns of occurrence on bark surface of living trees, leaf litter, and dung. Mycologia 81: 608-621. http://dx.doi.org/10.2307/ 3760136

Stephenson, S.L. \& Mitchell, D. 1994. Notes on tropical Myxomycetes. I. Collections from Ecuador and Peru. Micología Neotropical Aplicada 7: 17-21.

Stephenson, S.L., Schnittler, M. \& Lado, C. 2004. Ecological characterization of a tropical myxomycete assemblage - Maquipucuna Cloud Forest Reserve, Ecuador. Mycologia 96(3): 488-497. http://dx.doi.org/ $10.2307 / 3762169$

Stephenson, S.L., Kalyanasundaran, I. \& Lakhanpal, T.N. 1993. A biogeographical comparative study of myxomycetes in the mid-Appalachians in the esastern North America and two regions of India. Journal of Biogeography 20: 645-657. http://dx.doi.org/10.2307/2845520

Weberbauer, A. 1945. El mundo vegetal de los Andes peruanos [...] Nueva edición. Lima: Estación Experimental Agrícola de La Molina. Dirección de Agricultura. Ministerio de Agricultura.

Whitney, K.D. \& Keller, H.W. 1982. A new species of Badhamia, with notes on Physarum bogoriense. Mycologia 74(4): 619-624. http://dx.doi.org/ $10.2307 / 3792750$

Whitney, K.D. \& Olive, L.S. 1983. A new Didymium from Rarotonga. Cook Islands. Mycologia 75(4): 628-633. http://dx.doi.org/10.2307/3792992

Wrigley de Basanta, D. 2004. The effect of simulated acid rain on corticolous myxomycetes. Systematic and Geography of Plants 74: 175-181.

Wrigley de Basanta, D. \& Lado, C. 2005. A taxonomic evaluation of the stipitate Licea species. Fungal Diversity 20: 261-314.

Wrigley de Basanta, D., Lado, C. \& Estrada-Torres, A. 2008b) Morphology and life cycle of a new species of Didymium (Myxomycetes) from arid areas of Mexico. Mycologia 100(6): 921-929. http://dx.doi.org/ $10.3852 / 07-168$

Wrigley de Basanta, D., Lado, C. \& Estrada-Torres, A. 2010a) Licea eremophila, a new myxomycete from arid areas of South America. Mycologia 102(5): 1185-1192. http://dx.doi.org/10.3852/09-309

Wrigley de Basanta, D., Lado, C. \& Estrada-Torres, A. 2011. Spore to spore culture of Didymium operculatum, a new myxomycete from the Atacama Desert of Chile. Mycologia 103(4): 895-903. http://dx.doi. org/10.3852/10-363

Wrigley de Basanta, D., Lado, C. \& Estrada-Torres, A. 2012. Description and life cycle of a new Physarum (Myxomycetes) from the Atacama Desert in Chile. Mycologia 104(5): 1206-1212. http://dx.doi.org/ 10.3852/11-427

Wrigley de Basanta, D., Lado, C., Estrada-Torres, A. \& Stephenson, S.L. 2009. Description and life cycle of a new Didymium (Myxomycetes) from arid areas of Argentina and Chile. Mycologia 101(5): 707-716. http://dx.doi.org/10.3852/08-227 
Wrigley de Basanta, D., Lado, C., Estrada-Torres, A. \& Stephenson, S.L. 2010b) Biodiversity of myxomycetes in subantarctic forests of Patagonia and Tierra del Fuego, Argentina. Nova Hedwigia 90: 45-79. http:// dx.doi.org/10.1127/0029-5035/2010/0090-0045

Wrigley de Basanta, D., Lado, C., García-Martín, J.M. \& EstradaTorres, A. 2015. Didymium xerophilum, a new myxomycete from the tropical Andes. Mycologia 107(1): 157-168. http://dx.doi. org $/ 10.3852 / 14-058$

Wrigley de Basanta, D., Stephenson, S.L., Lado, C., Estrada-Torres, A. \& Nieves-Rivera, A.M. 2008a) Lianas as a microhabitat for myxomycetes in tropical forests. Fungal Diversity 28: 109-125.
Zar, J.H. 1996. Biostatistical analysis. Prentice Hall, Inc., Upper Saddle River, NJ.

Zúñiga de Acleto, R. 2010. Registro preliminar de Myxomycetos en la selva central del Perú. Biotempo 10: 15-17.

Associate Editor: Francisco Pando Received: 02-XII-2015 Accepted: 03-II-2016 